\title{
Evolution of Traffic Microsimulation and Its Use for Modeling Connected and Automated Vehicles
}

\author{
Narayana Raju (iD) and Haneen Farah (iD \\ Department of Transport and Planning, Faculty of Civil Engineering and Geosciences, Delft University of Technology, Stevinweg 1, \\ 2628 CN Delft, Netherlands \\ Correspondence should be addressed to Narayana Raju; s.s.n.raju@tudelft.nl
}

Received 14 April 2021; Revised 12 August 2021; Accepted 4 September 2021; Published 25 September 2021

Academic Editor: Peter J. Jin

Copyright (C) 2021 Narayana Raju and Haneen Farah. This is an open access article distributed under the Creative Commons Attribution License, which permits unrestricted use, distribution, and reproduction in any medium, provided the original work is properly cited.

\begin{abstract}
Traffic microsimulation has a functional role in understanding the traffic performance on the road network. This study originated with intent to understand traffic microsimulation and its use in modeling connected and automated vehicles (CAVs). Initially, the paper focuses on understanding the evolution of traffic microsimulation and on examining the various commercial and opensource simulation platforms available and their importance in traffic microsimulation studies. Following this, current autonomous vehicle (AV) microsimulation strategies are reviewed. From the review analysis, it is observed that AVs are modeled in traffic microsimulation with two sets of strategies. In the first set, the inbuilt models are used to replicate the driving behavior of AVs by adapting the models' parameters. In the second strategy, AV behavior is programmed with the help of externalities (e.g., Application Programming Interface (API)). Studies simulating AVs with inbuilt models used mostly VISSIM compared to other microsimulation platforms. In addition, the studies are heavily focused on AVs' penetration rate impact on traffic flow characteristics and traffic safety. On the other hand, studies which simulated AVs with externalities focused on the communication aspects for traffic management. Finally, the cosimulation strategies for simulating the CAVs are explored, and the ongoing research attempts are discussed. The present study identifies the limitations of present CAV microsimulation studies and proposes prospects and improvements in modeling AVs in traffic microsimulation.
\end{abstract}

\section{Introduction}

Automated vehicles (AVs) are closer than ever to making their mark over the road network. Given that AVs will ultimately not require the involvement of humans, which is known to be a contributing factor in most accidents [1], both researchers and practitioners have great hopes that AVs will improve the performance characteristics of traffic and enhance its safety standards $[2,3]$. However, verifying the assertions about AVs' added benefits to traffic efficiency and safety is quite challenging. Traffic microsimulation (i.e., soft computing platform for modeling the detailed intervehicular interactions in a traffic stream) can help to understand the implications of AVs' performance and communication aspects on traffic and support developing better traffic management strategies. Considering this, traffic microsimulation and its application to understanding the implications of AVs is vital. Therefore, this paper first describes the evolution of traffic microsimulation tools and then reviews the state-ofthe-art in using traffic microsimulation for modeling the implications of Connected and Automated Vehicles (CAVs) on traffic efficiency and safety.

The following sections include an introduction to traffic microsimulation (Section 1.1), followed by an introduction to CAVs (Section 1.2) and finally identifying the research aim and method (Section 1.3).

1.1. Traffic Microsimulation. For setting the traffic signals, Webster [4] attempted a computer simulation technique to support the framework in developing optimum signal time for the intersections. This can be marked as one of the initial attempts in traffic microsimulation. To account for the randomness in traffic modeling, researchers have relied on 
Monte Carlo simulation techniques [5-7] and cellular automata models [8, 9]. Simultaneously, researchers have developed and integrated mathematical models, such as different car following models [10] and lane changing models [11], into different simulation tools [12, 13]. The basic functionality of the simulation is time and space, along with the system state. Both time and space can be categorized as discrete or continuous with a system state. In this direction, a series of simulation approaches have been developed over time. In microsimulation, models exhibit discrete-time and continuous space phenomenon.

Advancements in computing and developments of new mathematical frameworks have led to major upgrades to microsimulation packages. Supported by advanced processing in computations, traffic microsimulation models are gradually found to be meticulous and realistic. Relying on empirical traffic data for analysis may not be feasible at all times, and implementation of proposed solutions to the traffic network without any checks can put human life at risk and cause unnecessary damage to the infrastructure. Traffic microsimulation offers numerous customization options for testing a variety of scenarios. Sensing the importance of microsimulation in understanding traffic characteristics, researchers have conducted numerous studies and developed various traffic microsimulation frameworks over the last few decades. These studies relate to various aspects of traffic, including among others car following [14], lane changing [15], signal control [16], traffic safety [17], traffic emissions [18], pedestrian flow [19], ramp metering [20, 21], roundabouts [22], toll operations [23], and truck parking operations [24]. To visualize this, the literature related to traffic microsimulation was identified by Scopus [25] search engine using the keyword "traffic microsimulation" and was then segregated into subareas resulting in the network plot shown in Figure 1. The size of the nodes and thickness of the links in Figure 1 depict the scale of the publications in that area, and the color depicts the clusters.

Figure 1 shows that traffic microsimulation was utilized in all kinds of traffic studies, and numerous traffic flow modeling concepts were evolved in connection with microsimulation. Given the ease and effectiveness, both researchers and industry partners have extensively used microsimulation approaches in supporting their decision making. Furthermore, numerous researchers [26-30] strongly advocated the importance of calibration for the reliability of the microsimulation outcomes. It is observed that researchers of various backgrounds widely use microsimulation in their studies. New microsimulation platforms were also developed and upgraded over time with the purpose of commercialization. Traffic microsimulation platforms have found a place across various domains for searching for optimized solutions.

1.2. Connected and Automated Vehicles. Researchers from both academic and industrial backgrounds, over the last five decades, believed that automobiles will achieve the selfdriving functionality and evolve as autonomous vehicles [31-33]. Given the advancements in computing and the availability of superior complex modeling, research on AVs has progressed. Currently, most of the automobile industries focus on developing their own AVs. Considering the potential market for AVs, multinational conglomerates showed a great interest in developing AVs [34]. To harmonize the definition of the level of automation, the Society of Automotive Engineers (SAE) has proposed six levels of autonomy, ranging from level 0 (no driving automation) to level 5 (full automation). The SAE levels of automation primarily differ in their Dynamic Driving Tasks (DDTs), Object and Event Detection and Response (OEDR), and Operational Design Domain (ODD). The ODD is the conditions under which Automated Driving Systems (ADSs) are designed to work. At level 0 , drivers will perform all the DDTs and OEDR. At level 1, drivers will still perform some of the DDTs and be responsible for the OEDR. At level 2, the system will perform the lateral and longitudinal DDTs while the drivers will be still responsible for the OEDR. At higher levels, the system will be responsible for the DDTs and the OEDR. The ODD of levels 1 to 4 is limited. At level 5 automation, unconditional DDTs will be carried by the ADS and unlimited ODD.

Past and ongoing research attempts have resulted in developing AVs with initial SAE level functionalities. Simultaneously, to assess AVs driving performance, AVs are being tested at various testbed conditions. Several researchers [35-37] strongly advocate that AVs can significantly impact the traffic characteristics of the road network. Unlike regular traffic, empirical data on AVs in traffic are still relatively scarce, and field experiments are limited given the risk for human life and potential damage to the infrastructure. Considering this, traffic microsimulation can play a huge role in assessing AVs impacts on the road network.

1.3. Research Aim and Method. The main aim of this paper is to understand the development of the research on traffic microsimulation in connection with modeling AVs. To achieve this aim, this study was carried out in two stages:

(i) Firstly, considering the significance of traffic microsimulation platforms in simulation studies, the progression of traffic microsimulation and microsimulation platforms were reviewed.

(ii) Secondly, the modeling aspects of AVs in traffic microsimulation and cosimulation with AV simulation tools were reviewed.

In the present study, literature was mined in three different parts: traffic microsimulation, C/AV microsimulation, and cosimulation studies. To understand the trend of traffic microsimulation, literature related to traffic microsimulation was first identified and then segregated using the keywords reported in Table 1 and the Scopus [25] search engine. Scopus database contains only the publications with Scopus indexing. The Scopus search engine provides advanced search options like authors, keywords, title, affiliation, journal title, avoid references, along with different conditional options for sorting the search. The keywords that were used for identifying relevant traffic 


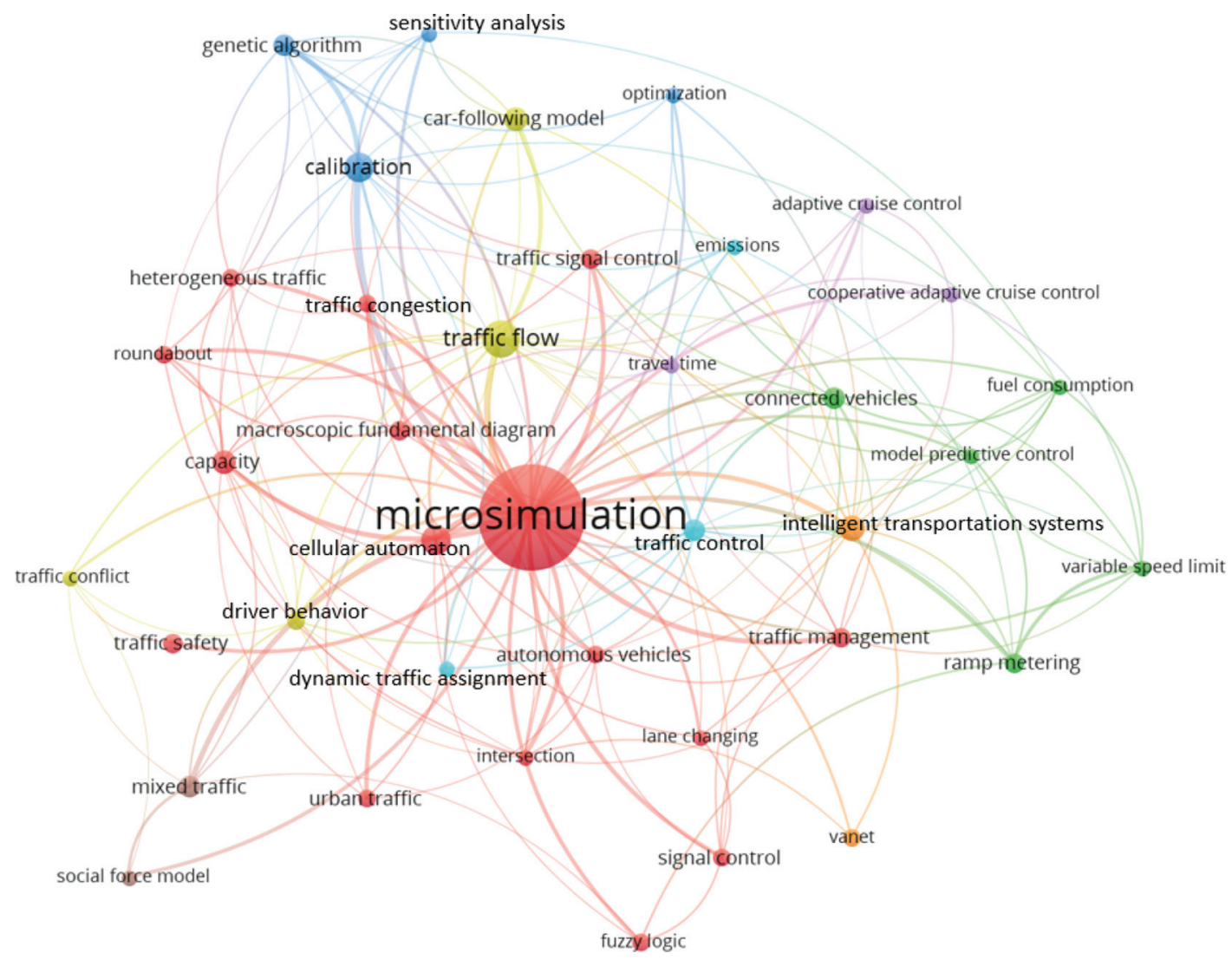

FIGURE 1: Keyword network from traffic microsimulation studies.

TABle 1: Adopted nomenclature in the study.

\begin{tabular}{|c|c|}
\hline Adopted words & Similar words in the literature \\
\hline Microsimulation & $\begin{array}{l}\text { Microscopic simulation, microlevel simulation, microscopic traffic simulation, microlevel traffic simulation, } \\
\text { simulation, traffic simulation, vehicle simulation }\end{array}$ \\
\hline $\begin{array}{l}\text { Autonomous vehicles } \\
\text { (AVs) }\end{array}$ & $\begin{array}{l}\text { Self-driving vehicles, autonomous cars, automated cars, automated vehicles, robotic vehicles, autonomous } \\
\text { unmanned surface vehicle, https://acronyms.thefreedictionary.com/Autonomous+Unmanned+Vehicle }\end{array}$ \\
\hline Driving behavior & $\begin{array}{c}\text { Driver behavior, vehicular behavior, vehicle behavior, (multi-)agent behavior, (multi-)agent trajectory, lane } \\
\text { changing, merging }\end{array}$ \\
\hline Traffic flow & Traffic stream, stream flow, network flow \\
\hline Traffic safety & Road safety, driver safety, safety \\
\hline Emissions & Emission modeling, traffic emission, vehicular emission \\
\hline Traffic management & Vehicle management, network management \\
\hline
\end{tabular}

microsimulation papers were microscopic simulation, microlevel simulation, microscopic traffic simulation, microlevel traffic simulation, simulation, traffic simulation, and vehicle simulation. Initially, articles were checked individually for keywords, titles, and abstracts. In the next phase, for a given search keyword, all the articles were sorted, and duplicates were eliminated. Further, from the sorted articles, each article was checked by the authors; this check included the title of the work, followed by the abstract (in this phase the authors marked the microsimulation studies and platforms); finally, for the unclear articles, the research methodology of each article was examined. On this basis, the authors identified the articles in the domain of microsimulation.

Literature related to $\mathrm{C} / \mathrm{AVs}$ and cosimulation was mined using Scopus [25], Google Scholar (2021), and
Mendeley (2021) databases. Google Scholar is a basic search platform, where the search engine focuses on the article title and the abstract for the search word. Further, those articles can be added to the library and exported as .csv files for other analyses. Like Google Scholar, Mendeley offers similar functions based on its search engine. Both Google Scholar and Mendeley contain all kinds of publications.

From the literature review, it was observed that researchers used different nomenclature in describing the same term. For example, some studies reported traffic microsimulation as traffic microscopic simulation, microlevel simulation, and microsimulation. To limit this lack of consistency, similar words were clustered into a unique word, as reported in Table 1. 
The rest of the paper is structured as follows: Section 2 presents the sources of traffic microsimulation studies, followed by the review of different microsimulation platforms in Section 3. Section 4 synthesizes the AVs traffic microsimulation studies, and Sections 5 and 6 summarizes the paper and introduces the prospects and future research directions for AVs traffic microsimulation.

\section{Sources of Traffic Microsimulation Studies}

Researchers across the world have used traffic microsimulation to understand the traffic characteristics, such as driving behavior, traffic management, and traffic safety. Traffic characteristics are varying in nature with different geographical, cultural, and economic conditions. For example, vehicles have proper lane discipline under homogeneous traffic conditions. In the case of cold climatic conditions, cars have dominant proportion in comparison to motorized two-wheelers. On the other hand, in Southeast Asia and South Asian countries, given the favorable conditions with added fuel mileage, the proportion of motorized two-wheelers is higher. Along similar lines, the physical characteristics of vehicles are varying in nature across the world. Furthermore, economic status of countries affects their advancements in transportation infrastructure. Differences in cultures and norms affect road users' behavior. Road user behavior, which is the root source of understanding traffic performance, is highly diverse with different circumstances. As a result, solutions to traffic problems are never taking a unique form.

To understand the geographical distribution of traffic microsimulation studies, the identified literature was mapped considering author's affiliations and nationality. This would help in understanding the quantum of microsimulation studies sources. For visualization purposes, a radial plot is presented in Figure 2. The rectangular bars represent the number of publications for each affiliation. It can be observed that traffic microsimulation studies were conducted all over the world, with a significant share by the United States of America (27\%), China (26\%), Germany (7\%), and Netherlands (7\%). Some of the major universities in these countries have contributed heavily to this research domain: in Netherlands, Delft University of Technology (211); in China, Tongji University (96), Beijing Jiaotong University (96), Southeast University Nanjing (80), and Tsinghua University (63); in the United States of America, Virginia Polytechnic Institute and State University (79) and University of California, Berkeley (57); in Germany, Technical University of Munich (56); and in Singapore, the National University of Singapore (51). In recent years, in addition to developed nations, microsimulation studies are slowly picking up as well in developing nations.

In the present context, around 1250 traffic microsimulation studies have been published in more than 50 scientific journals. The analysis was carried forward to understand the articles' share in journals as shown in Figure 3. Note that in Figure 3, only journals with a minimum of 15 published articles were considered. The Transportation Research Record (28\%) has the largest share, followed by Transportation Research Part C: Emerging Technologies (10\%), IEEE Transactions on Intelligent Transportation Systems (7\%), and the Journal of Transportation Engineering (6\%). Publications in other journals with smaller share are also presented in Figure 3. With respect to conference proceedings, IEEE International Conference on Intelligent Transportation Systems (ITSC) proceedings (177) and Transportation Research Procedia (80) have accumulated most studies in this domain.

\section{Traffic Microsimulation Platforms}

With the increase in computational efficiency and availability, researchers and industry partners used programming scripts and input parameters to integrate the traffic modeling concepts into simulation packages. Initially, simulation packages were limited to case-specific ones with a small set of programming scripts. Most of the simulation platforms in their early versions were basic, including the car following functionality on a straight midblock road section. Given the opportunities with the evolution in programming over the last three decades, developers and researchers have updated different microsimulation platforms. In addition, the evolution of new research concepts, such as car following and lane changing models and traffic signal optimization techniques, led to various inbuilt customization options in traffic simulation over time. To understand these better, present traffic microsimulation platforms were reviewed, and their details and characteristics are summarized in Table 2.

Paramics [38] project was supported by the UK Department of Transportation and developed by the researchers of the University of Edinburgh, Scotland. The initial platform was purely based on the Hans-Thomas Fritzsche car following model [39]. Later, research inputs from the University of California, Irvine, helped in embedding numerous packages, which include traffic signals, ramp metering, and bottleneck in freeway scenarios. Finally, in the year 2005, the Quadstone Paramics acquired the Paramics simulation platform. On the other hand, another variant of Paramics, S-Paramics, was distributed by the SIAS Group [40]. Presently, both Quadstone Paramics and S-Paramics are identified as old and new Paramics, respectively. Like Paramics, VISSIM was developed by researchers of Karlsruhe University, Germany, with a psychophysical car following model [41] and other input packages which were added over time. Later, PTV group acquired VISSIM from Karlsruhe University and developed it further as PTV VISSIM. Under MIT's Intelligent Transportation Systems (ITS) program in collaboration with Caliper Corporation, researchers of the Massachusetts Institute of Technology, USA, developed MITSIM $[42,43]$ with the idea of an open-source traffic microsimulation.

There are many other platforms including AIMSUN [44], MIXIC [45], CORSIM [46, 47], SUMO [48, 49], TransModeler [50, 51], MOTUS [52, 53], TRITONE [28], and OpenTrafficSim [54]. Among these platforms, AIMSUN, CORSIM, and TransModeler are commercial, whereas SUMO, MOTUS, TRITONE, and OpenTrafficSim are open- 


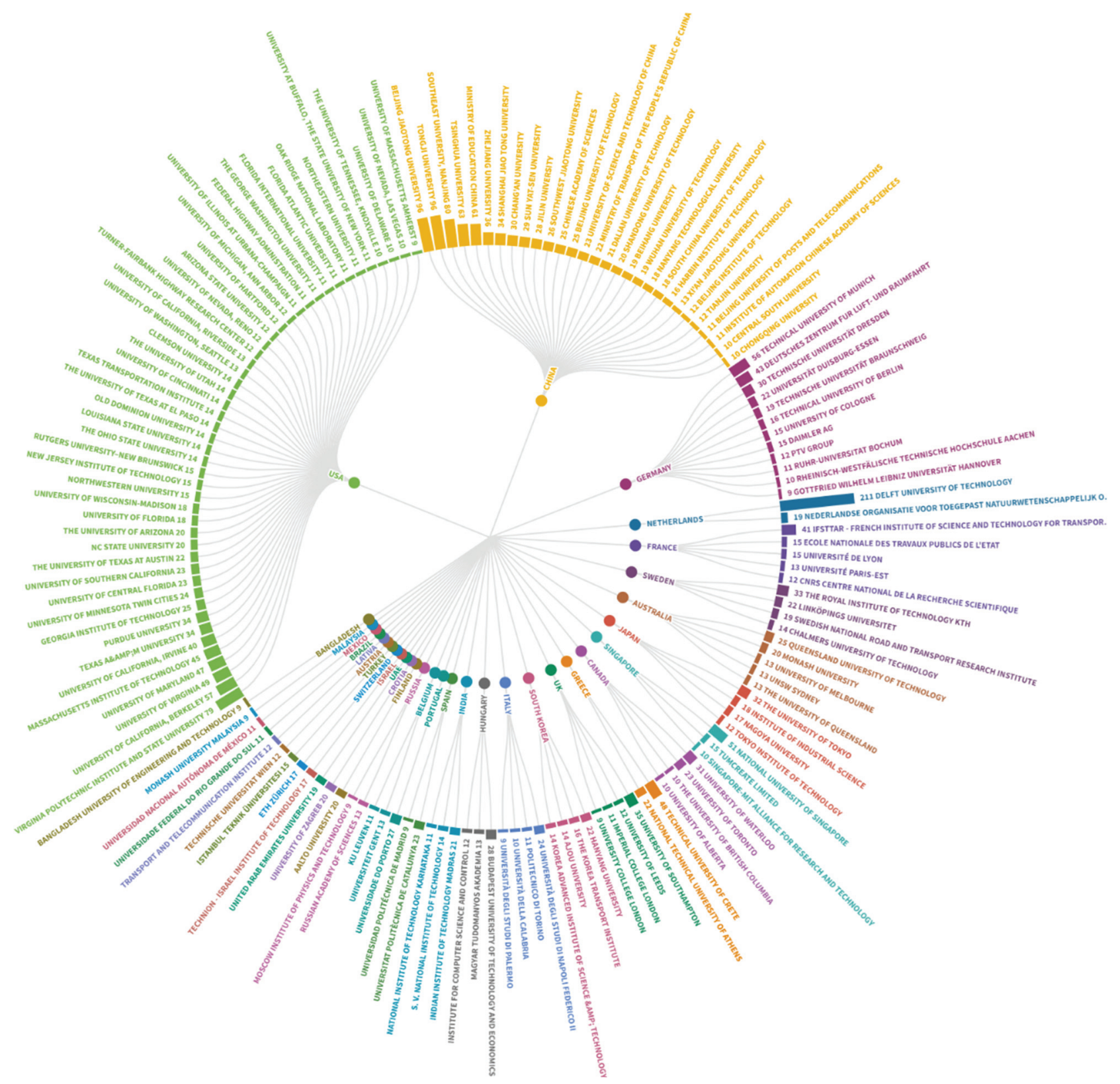

FIgURE 2: Radial plot depicting the involved institutes across the nations.

source platforms. Other microsimulation platforms, such as Integration [55], Sim Traffic [56], HUTSIM [57], THOREAU [58], FAUSIM [59], FOSIM [60], RuTSIM [61], and GLD traffic simulator [62], evolved from certain project ideas. It can be noted that most of the established microsimulation platforms were instigated in the 90s and were developed with the idea of simulating homogeneous lane-based traffic conditions.

From the review of the different simulation packages, it is observed that most of traffic microsimulation platforms were initiated and developed by transportation researchers at their institutes within frameworks of specific projects. Later, in collaboration with industrial partners, these simulation platforms have further evolved, which led to the development of commercial traffic microsimulation platforms. On the other hand, some traffic microsimulation platforms were kept open in carrying the research. Most commercial variants provide free time-limited licenses for academics. Microsimulation platforms with explicitly available details are summarized in Table 2.

Further, based on the specific functionality (core behavioral models, optimizing computation, user groups), microsimulation platforms were developed over various programming interfaces. Most of the inbuilt models in simulation platforms are sufficient to test basic scenarios. On the other hand, in some cases, inbuilt models must be replaced by external behavioral logic/algorithms for the research analysis. Keeping this in mind, presently, external modules are offered in coding behavioral logic/algorithms. Those external modules give opportunities for researchers 


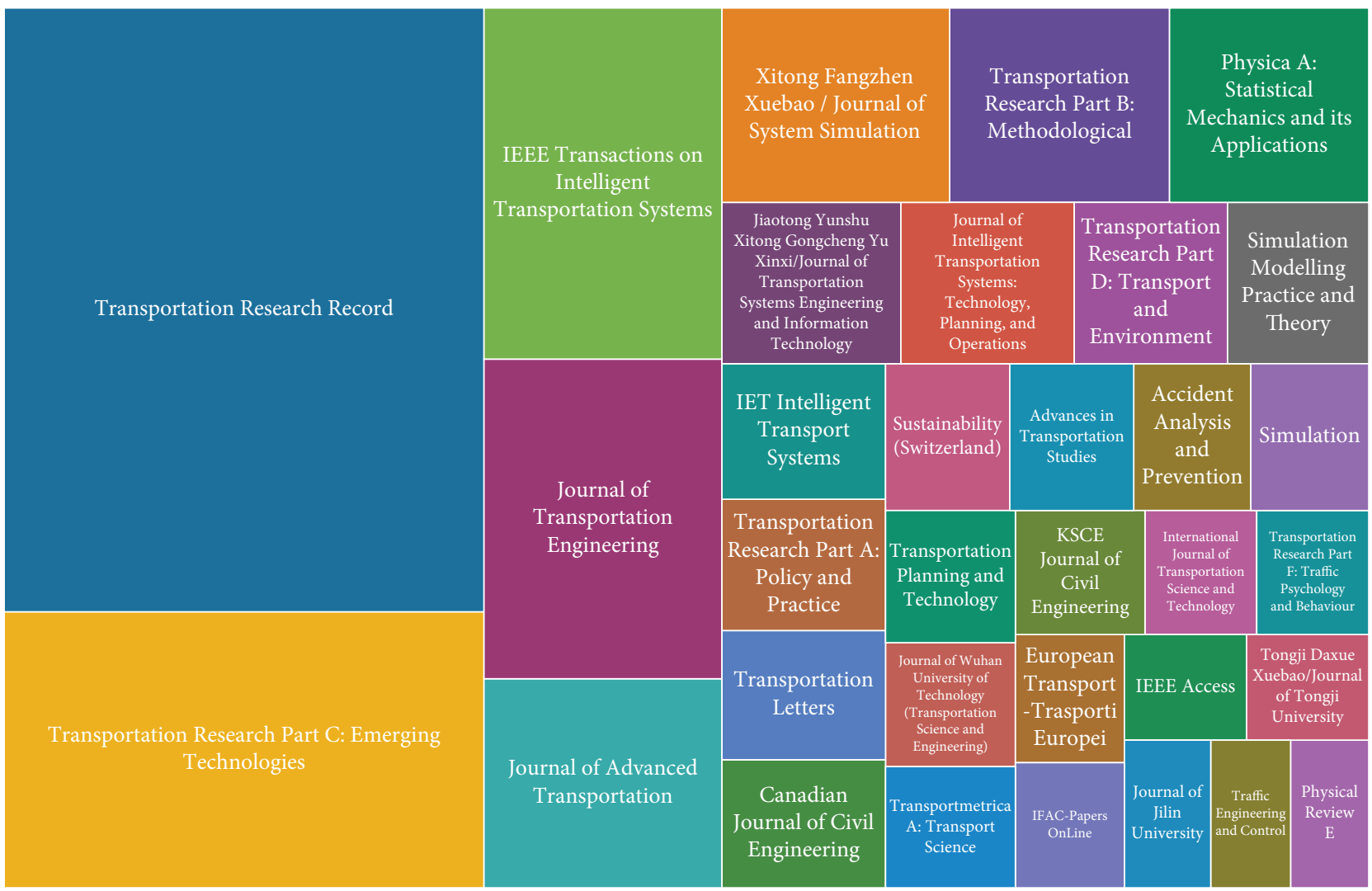

FIgURE 3: Traffic microsimulation work share of the journals with at least 15 papers.

to bypass the inbuilt logic. Various programming interfaces are used in different microsimulation platforms as detailed in Table 2. However, unlike microsimulation platforms with inbuilt models, the processing speed with external modules is lower; this again depends on the coded script's logical flow.

\subsection{Publication Share of Microsimulation Platforms. To} better understand the existing research using different microsimulation platforms, literature on traffic microsimulation platforms was mined. The identified studies were later segregated by the year of publication. A Mekko chart was prepared, as shown in Figure 4, to illustrate the results. The $x$-axis in Figure 4 represents the timeline in years, and the $y$-axis demonstrates the percentage share. The width of the rectangles depicts the scale of publications in that year. From the database, it is shown that initially the research work was limited to its developers. Later in early 2000s, those microsimulation platforms were open to researchers outside the development teams.

The number of publications was low initially, with a minimum of 17 publications in 2001, but gradually increased over the years to a record of a maximum of 376 publications in the year 2019. Unlike the increase in total publications, the share of the different microsimulation packages in these publications has varied over the years. Starting from 2001, CORSIM and Paramics have a fair share of the scientific publications whereas VISSIM and AIMSUN have a minor share. However, over time the shares of VISSIM and SUMO have increased, and in 2020 both had the biggest shares among other simulation platforms.

On the other hand, AIMSUN has a relatively uniform number of publications over the years. Publications using TransModeler have been relatively stable with a relatively minor share. Publications from MITSIM, MOTUS, MIXIC, TRITONE, HUTSIM, Integration, DRACULA, SimTraffic, and OpenTrafficSim have been identified over time, with most of the publications being from developers' backgrounds.

This analysis signifies that microsimulation in traffic engineering research has picked up the pace in the previous two decades. Numerous researchers and industry partners are testing different logic and scenarios using microsimulation platforms for decision making in the present driving context. Simultaneously the variation in the platforms' share can be attributed to various factors, including commercialization, user interface, supported traffic conditions, and user support.

Based on the number of publications in traffic microsimulation, the top twenty-five publishers were identified. Later, the publications were labeled based on the utilized microsimulation platform. To express this better, a Sankey plot was generated considering the microsimulation platforms and the journals, as shown in Figure 5. The width of the connection demonstrates the number of publications 


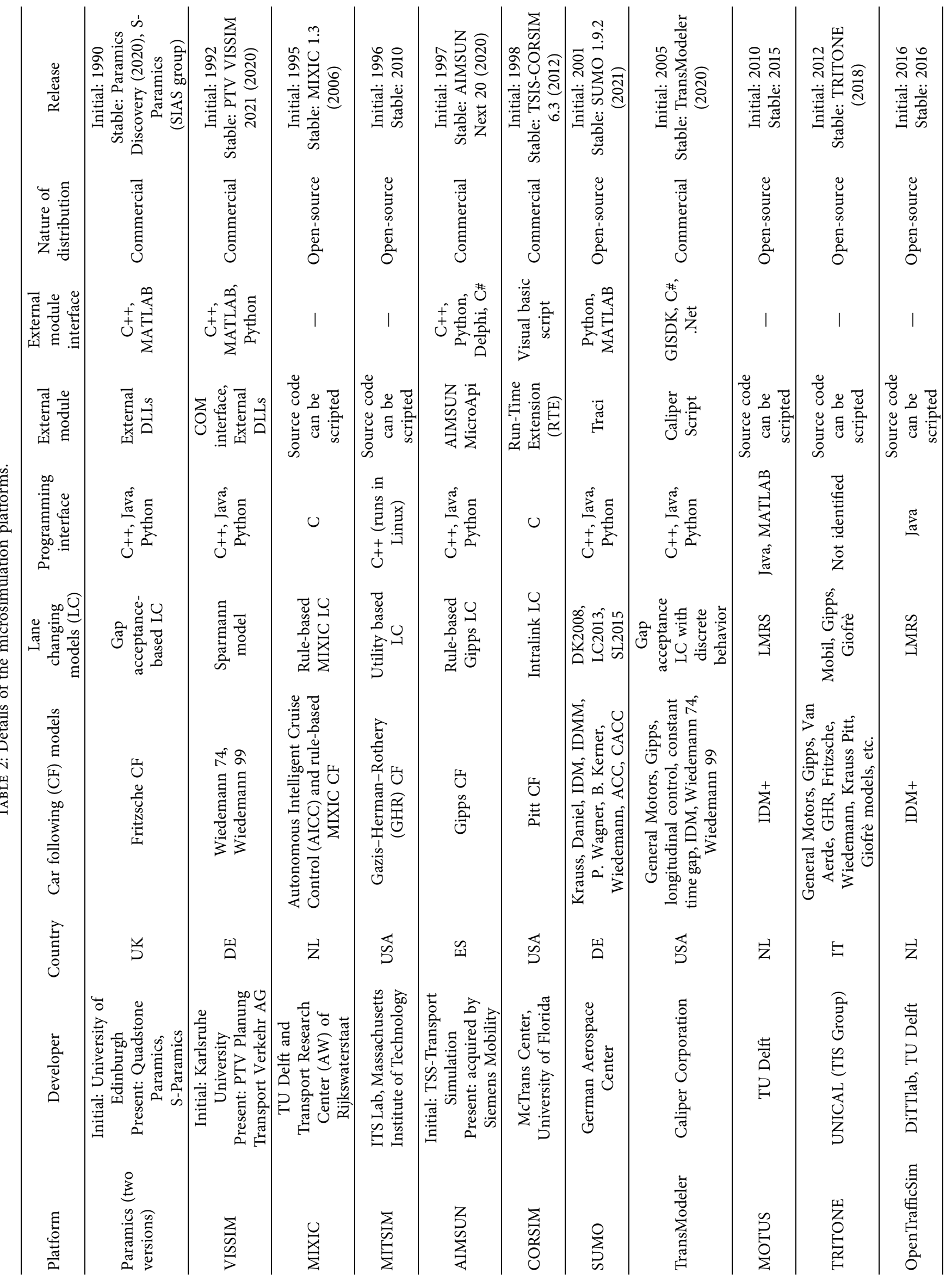




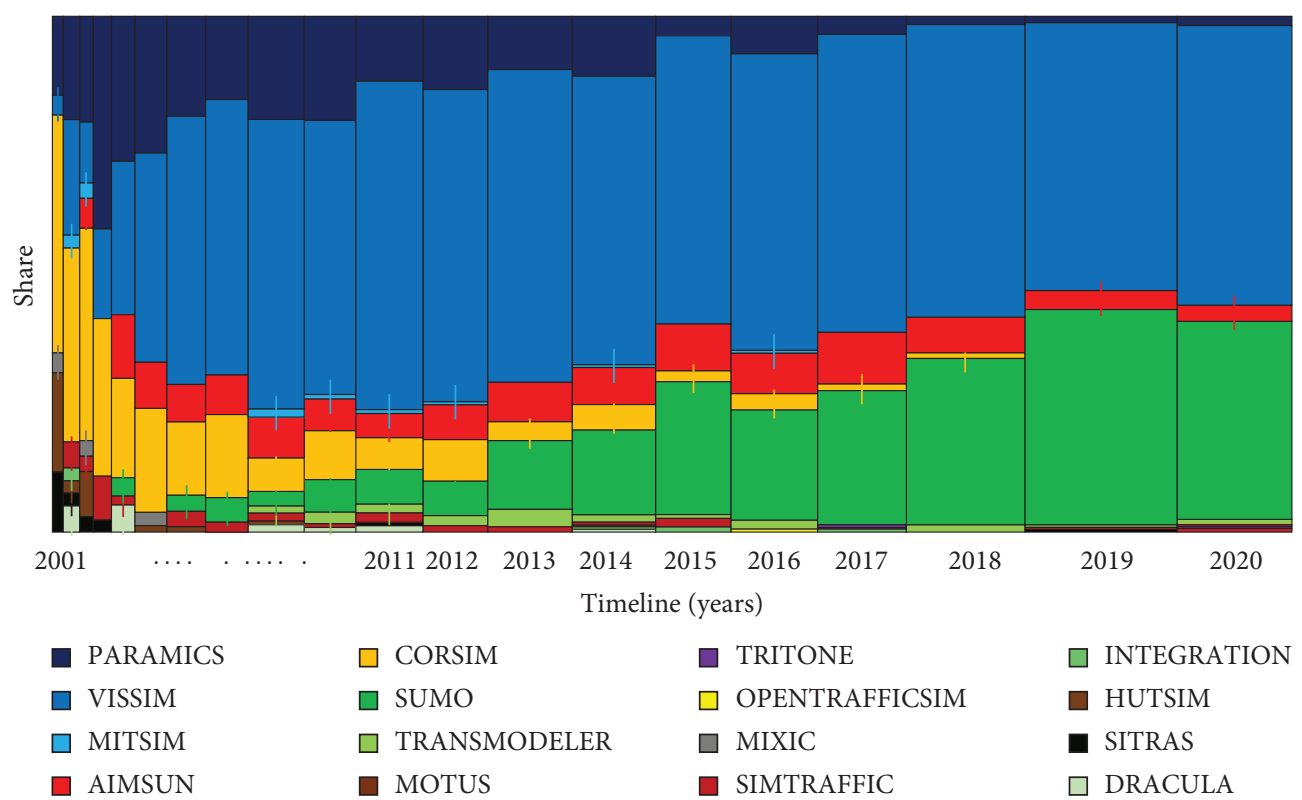

FIgUre 4: Publication share of microsimulation platforms over the years.

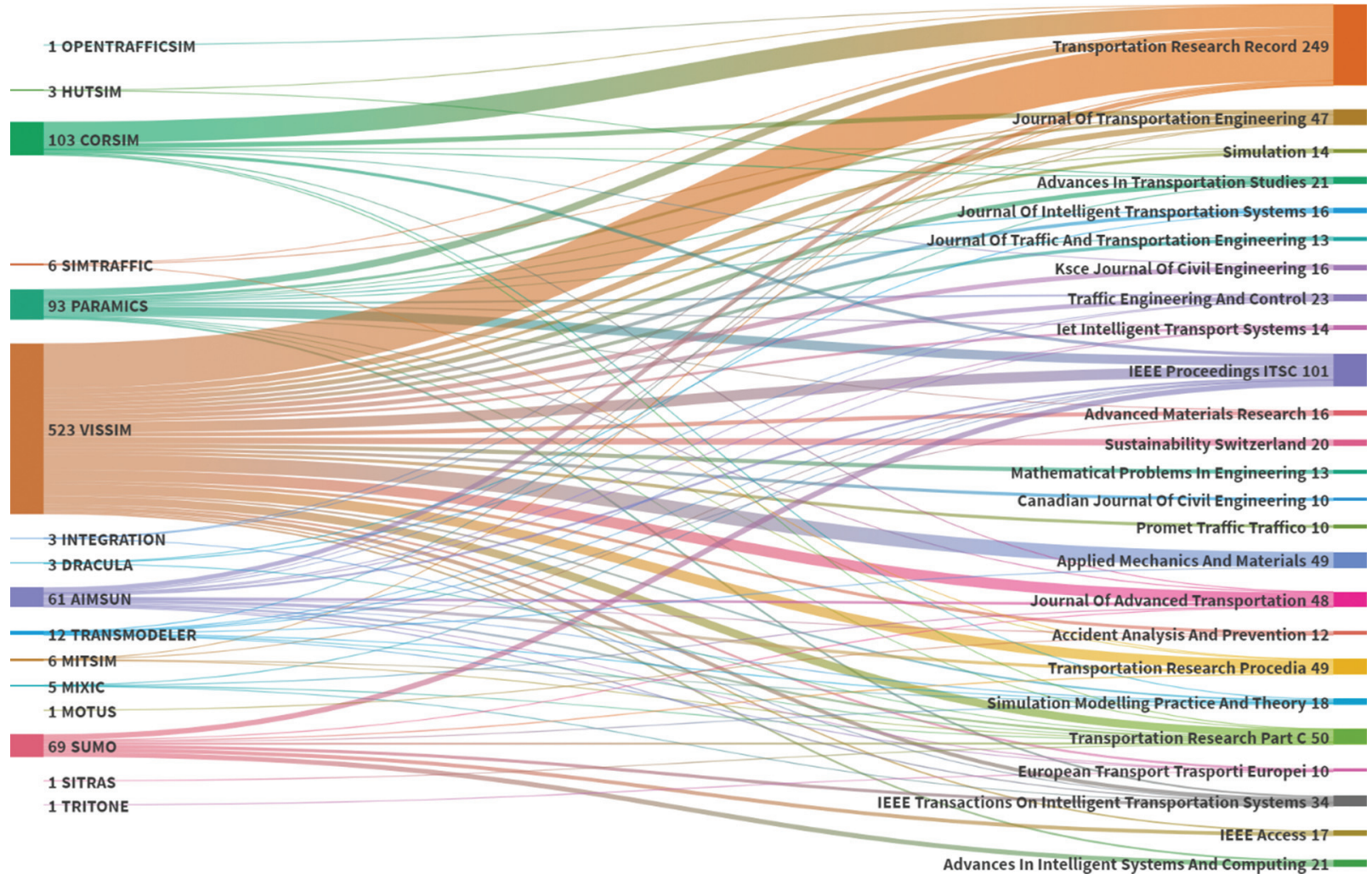

Figure 5: Publication shares of microsimulation platforms over top 25 publishers.

concerning the microsimulation package in that journal. The sizes of the head (microsimulation platform) and tail (publisher) nodes show the scale of publications. The analysis shows that Transportation Research Record, Transportation Research Part C, Journal of Transportation Engineering Part A, IEEE ITSC proceedings, Transport
Research Procedia, and Applied Mechanics and Materials have a significant share of microsimulation publications. It is noticed that, with 523 publications, VISSIM has a major share in all journals. CORSIM (103), Paramics (93), SUMO (69), and AIMSUN (58) had also a relatively significant share in publications. 


\section{Automated Vehicle Microsimulation}

Over the years, traffic microsimulation platforms have proven their mantle and their extensive use for various research and industrial activities across different domains. Presently traffic microsimulation platforms have reached a level that can support decision making of practitioners. At the same time, researchers and industrial partners are foreseeing the deployment of AVs on the exiting road network in the near future. Researchers of various backgrounds are predicting the use of AVs for private transport [63], public transport [64], and freight transport such as autonomous trucks [65], as well as for ridesharing [66] such as autonomous shuttles [67]. An increasing number of research studies $[68,69]$ support the claims that AVs improve traffic safety and efficiency. However, currently quantifying the impacts of AVs in real life from a traffic engineering point of view is highly challenging. This is because of several reasons, such as exposing road users to potential risks, uncertainty regarding the suitability of current traffic management plans, and road authorities being uncertain yet regarding the minimal infrastructure requirements for safe operation of AVs in mixed traffic. Simultaneously, developing strategies and frameworks from only an empirical, experimental point of view is challenging from a cost-benefit point of view. Therefore, the impact of AVs on traffic flow operation must be first studied extensively using traffic microsimulation. With proper utilization of technology and AVs' communication, a good quantum of benefits can be achieved from a traffic engineering point of view.

Given this potential of microscopic simulation platforms in carrying the research in this domain, researchers across the world have already performed various AV studies using different microscopic simulation platforms. Particularly, in the second half of the previous decade, these kinds of studies have picked up. To understand the research flow in this domain, keyword analysis was performed on the literature database, and the visualization plot in Figure 6 presents the findings. The size of the node and the thickness of the links in Figure 6 depict the scale of the work, and the colors depict different clusters. Similar to the regular traffic studies, AV microsimulation studies were also conducted in connection with many traffic related topics including ramp metering $[3,70]$, car following [71, 72], traffic signals [73], emissions [74], road safety [75], and mixed traffic [76].

Most of the microscopic simulation studies have differentiated AVs and human-driven vehicles by their driving behavior and inferred that the driving behavior is the crux in modeling AVs. In the present context, most researchers (e.g., $[77,78]$ ) unilaterally assumed that, in comparison to human-driven vehicles, the behavior of AVs is less stochastic. In addition, it is believed that AVs have good lane discipline and consistent behavior.

To investigate this in more detail, the literature was reviewed. From the initial assessment, it is observed that researchers used two main types of strategies to model AVs in simulation platforms. The first strategy is about bringing consistency in AVs driving behavior with the inbuilt car following and lane changing models. Following this direction, AV's behavior was regulated by adapting the model parameters. The second strategy is about coding AVs' behavior and connecting it to a simulation software using Application Programming Interface (API). In the following sections, these two approaches and the related studies are further described and discussed.

4.1. AV Modeling by Inbuilt Models. In giving due weightage to inbuilt behavioral models, researchers modeled AVs by adapting the parameters of the inbuilt car following models in microsimulation platforms. For example, to understand the safety at intersections and roundabouts due to AVs, $[79,80]$ adapted the Wiedemann model's behavior in VISSIM. It was found that AVs improve traffic safety and throughput of the study sections. With a similar framework, [81] modeled AVs at roundabouts. At a higher penetration rate of AVs, a $75 \%$ decrease in crash probability was observed compared to a base scenario with no AVs. Along similar lines, by adapting the psychophysical Wiedemann 74 and 99 car following models in VISSIM, various other studies were carried out for different test scenarios to understand the impacts of AVs. These studies included congested networks [82], traffic signals [83], roadway capacity [84], traffic stream characteristics [85], free speeds [86], operational performance [87], emission impacts [88], safety performance [89], unsignalized intersections [90, 91], and transitional impacts [92]. Other studies have adapted the inbuilt Krauss model in SUMO $[93,94]$ to model AVs. Zeidler et al. [95] tested the compatibility of inbuilt Wiedemann models for inducing the communication between $\mathrm{AVs}$ and compared it to field driven AVs. The focus of these mentioned studies, the behavioral models used, and the studies' key findings are summarized in Table 3.

It is observed that there is inconsistency in the findings of the studies regarding the penetration rates of C\AVs at which there is an improvement in safety and traffic efficiency, with reported penetration rates ranging from 20 to $70 \%$. Some studies demonstrated a deterioration in safety and drop in capacity standards at penetration rates below $30 \%$; this is observed in all kinds of modeled road sections. In addition, the transition point of the penetration rate of $\mathrm{AVs}$ at which an improvement is observed is not consistent across the studies.

The strategy of modeling AVs with inbuilt models has its advantages and disadvantages. When the inbuilt model parameters are tweaked for AVs, the microsimulation models typically run with fewer externalities; as a result, a consistent traffic movement will be observed at less computational capacity.

Many AV simulation studies with inbuilt models used VISSIM and relied on its psychophysical car following models. The influential critical parameters to mimic AVs vary among the studies and depend on the specific circumstances being studied as summarized in Table 3. It is inferred that very few studies considered the lane changing parameters for AV modeling. Furthermore, the modeled behavior of the AVs is highly oriented towards the inbuilt models; as a result, the simulated AVs can be less realistic in 


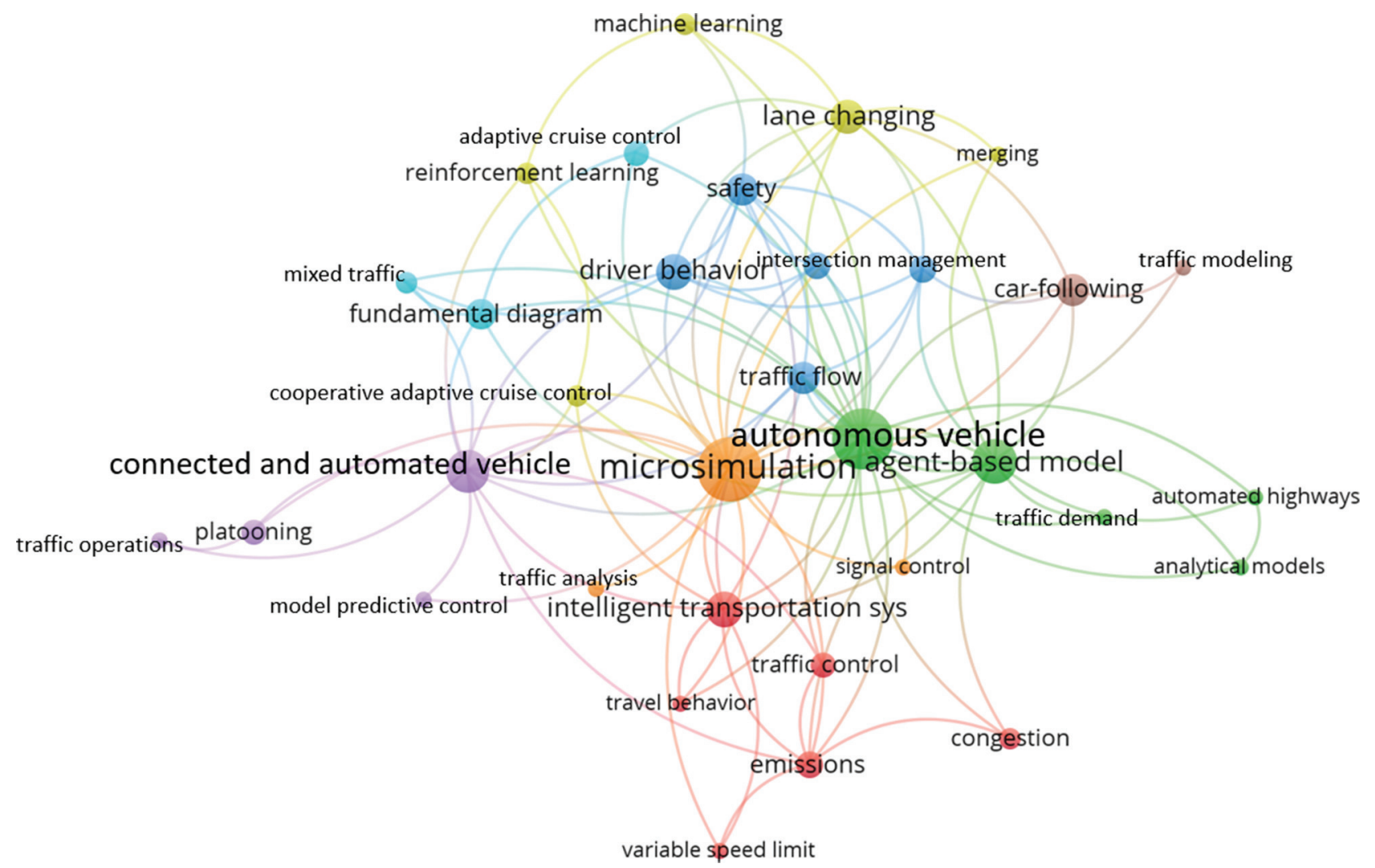

Figure 6: Keyword network of AV microsimulation studies.

comparison to field behavior. Furthermore, there seems to be less weightage to modeling the communication of AVs. Connectivity which is the key in CAVs was simulated by means of adapting the parameters that determine the headway and the number of observed vehicles, with any predefined logic/algorithms. Considering the importance of communication aspects of AVs, this is a huge limitation in this direction.

4.2. AV Modeling by Externalities. The externalities of microsimulation platforms give prospects in scripting the external logic. During the run-time of the simulation process and with the help of Application Programming Interface (API), the inbuilt models can be overridden, and as a result the vehicle motion can be dictated by the data feeds. This gives researchers a considerable advantage in testing their models/frameworks. Considering the potential of applying external models, researchers applied predefined behavior logic of AVs. Given the flexibility in controlling the characteristics of the simulation objects during the run-time, communication logic was also induced among the vehicles. The basic idea of this kind of studies is that AVs have the added functionality of communication as CAVs and are controlled by the externalities, while the human-driven vehicles are operated by the inbuilt models. Based on this framework, researchers modeled CAVs on different road segments and modeled various AV maneuvers. To understand this better, the literature related to $\mathrm{AV}$ simulation studies in connection with externalities has been reviewed and is summarized in Table 4 with details regarding the study focus, logic modeled, CAV behavior, and key findings.

It is observed that researchers applied numerous logic systems/algorithms, including adaptive dynamic programming [97], optimization-based ramp control strategy [70], Virdi CAV Control Protocol [99], decision-making CAV control algorithm [75], Model Predictive Control [100], Autonomous intersection management [107], Platooning Extension Plexe [108], cooperative scheduling mechanism for CAVs [110], discrete-time occupancies trajectory-based intersection traffic coordination algorithm [113], lane sorting [114], matrix-based intersection management logic [116], and Cooperative Controller and Distributed Algorithm [72].

The main aim of the algorithms mentioned above was to induce CAVs' behavior and instigate communication among the vehicles for better traffic movement over the traffic network. Researchers tested the CAVs across various traffic facilities, including midblock sections, signalized intersections, unsignalized intersections, on- and off-ramps, etc. The behavior of the AVs was governed by various models including the Gipps model [119], Intelligent Driver Model (IDM) [120], Optimum Velocity Model (OVM) [121], Adaptive Cruise Control (ACC) [45], Cooperative Adaptive Cruise Control CACC [71], Theoretical model, and cooperative longitudinal following behavior.

The focus of the researchers varied following the type of study segments. In midblock sections, researchers were 
Journal of Advanced Transportation

11

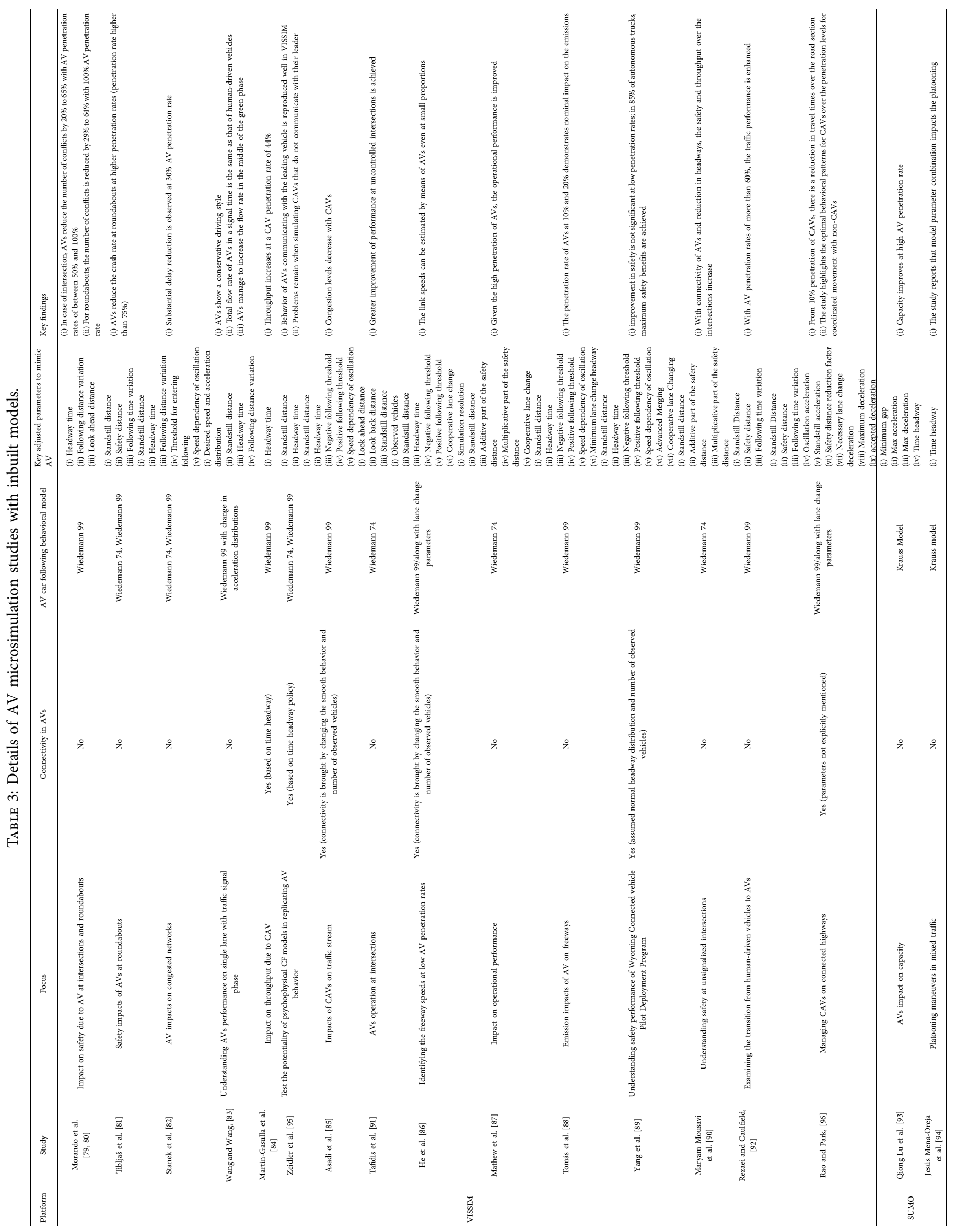




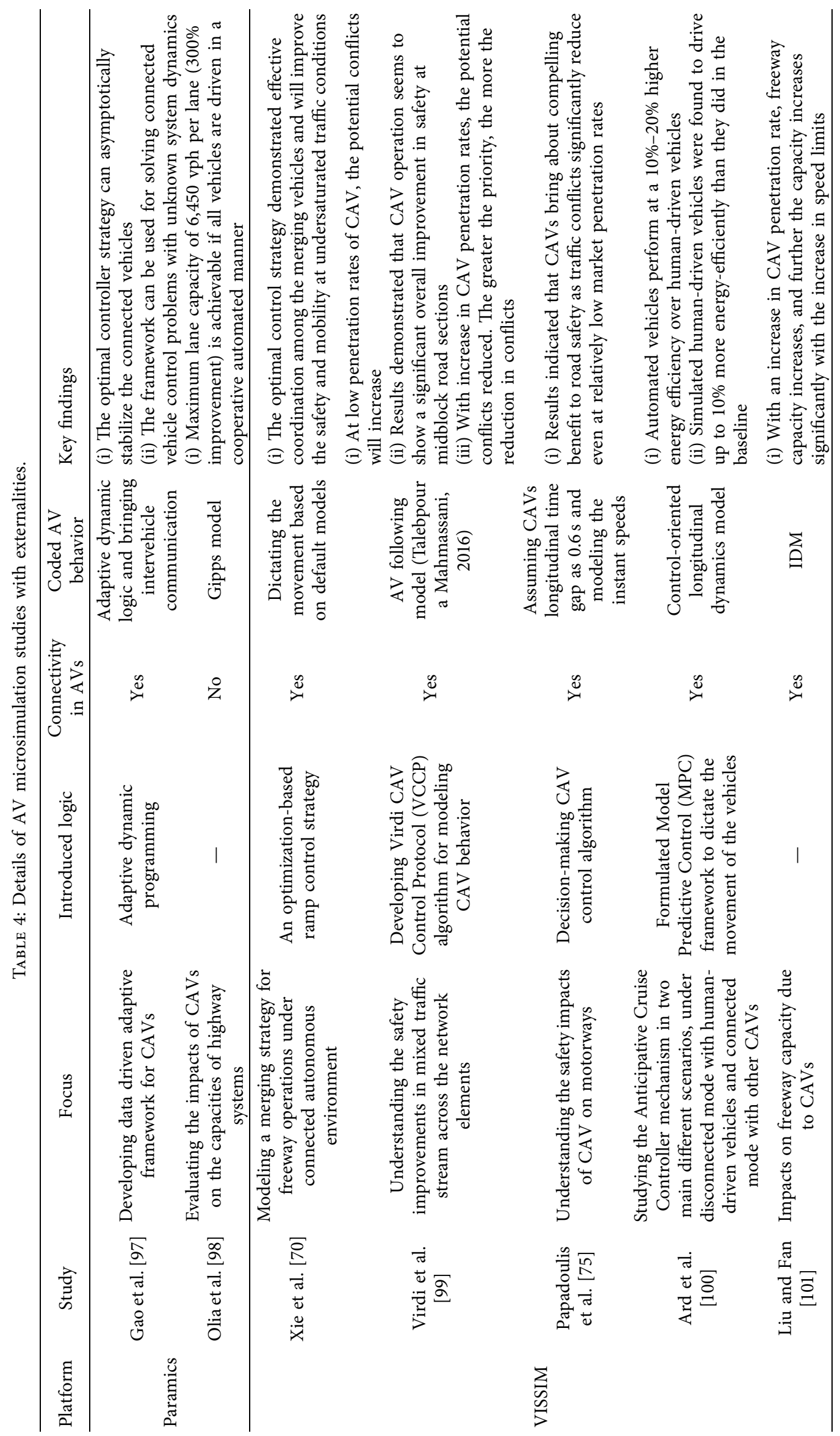




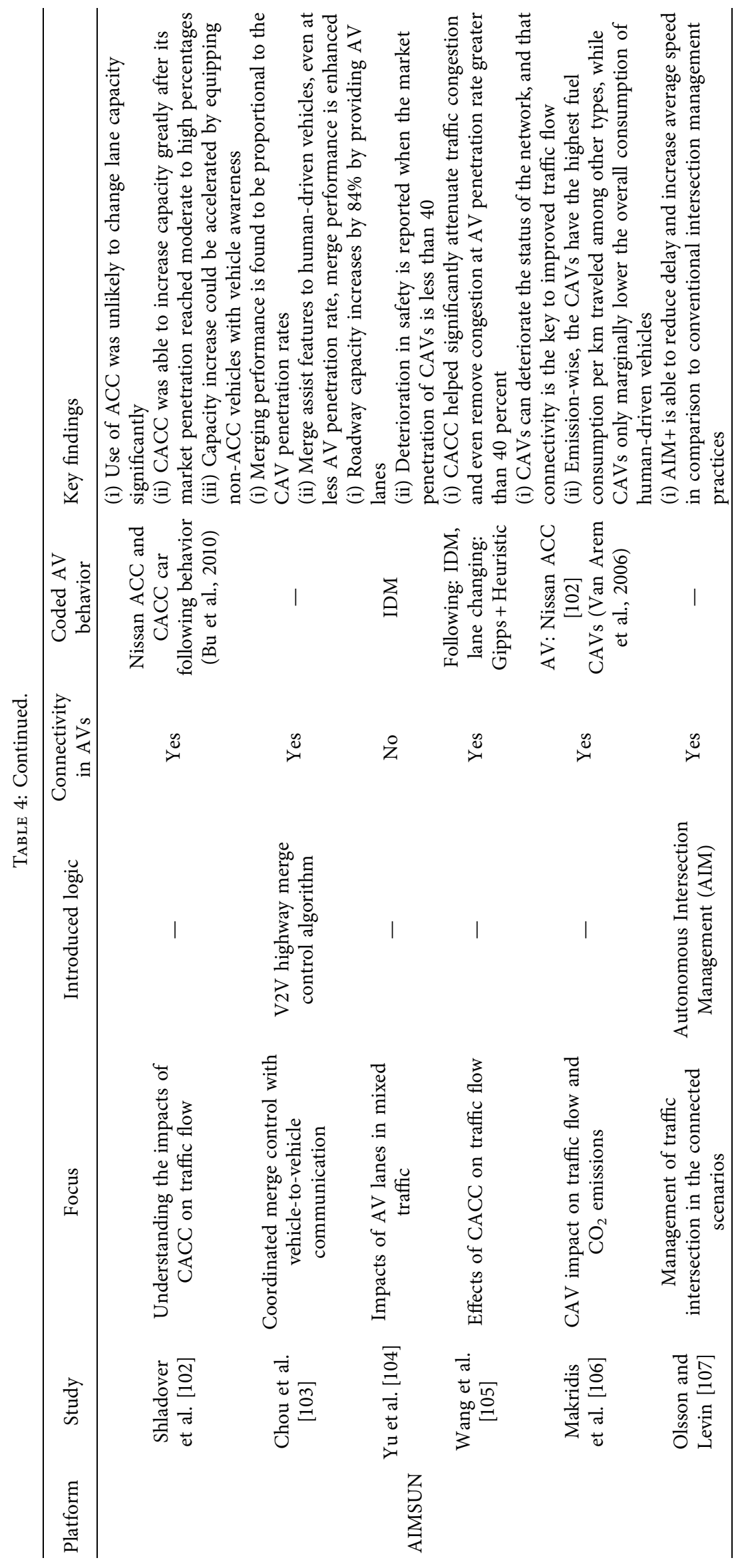




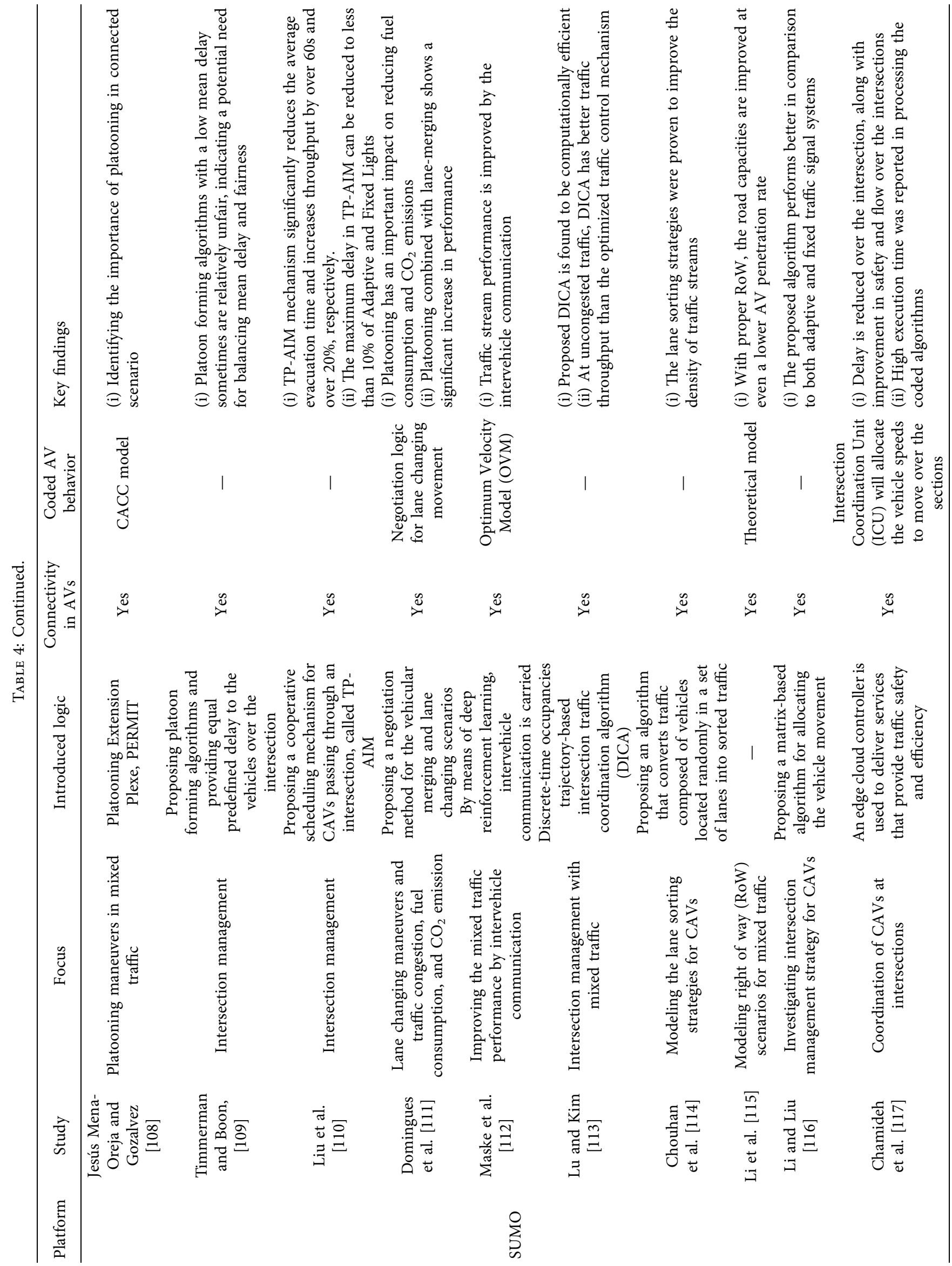




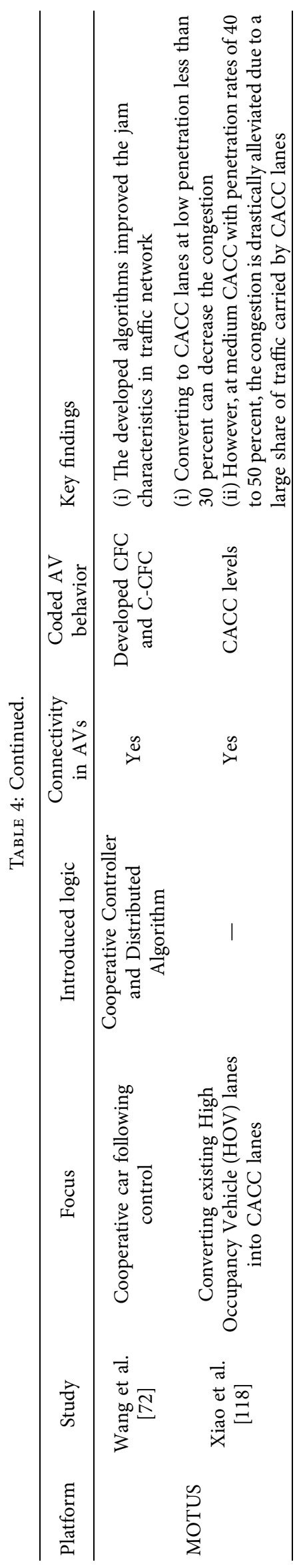


focused on CAVs following behavior and lane changing behavior with platooning logic to maximize the improvement in safety and throughput. On the other hand, at intersections and ramps, studies focused on communication among CAVs so that CAVs coordinate themselves and create necessary gaps for traffic movement, with the idea of limiting the stop and go conditions and eliminating traffic signals. Unlike the studies of CAVs with inbuilt models, the studies were focused on the efficacy of the behavioral logic rather than studying the penetration impacts of CAVs. To a certain extent, most of these studies demonstrated a good efficacy of their logic/algorithms. With some externalities, microsimulation platforms such as VISSIM, Paramics, and AIMSUN performed the simulation runs with a single core in the computer processor. As a result, the processing time with those externalities was high. Therefore, the application to a bigger network will be computationally inefficient. Furthermore, most of the logic/algorithms were tested at isolated study segments; therefore, their performance must be quantified in future studies for a traffic network level with greater stochasticity. Finally, to express the reviewed literature in a better manner, visualization analysis was carried out as shown in Figure 7.

4.3. Cosimulation. While in traffic microsimulation, interactions among vehicles are modeled and traffic characteristics are quantified, traffic microsimulation tools do not offer an opportunity to model the C/AV mechanism, and the behavior of the C/AVs is mainly governed by the behavioral models rather than the accurate perception of the infrastructure environment. On the other hand, from an individual $\mathrm{C} / \mathrm{AV}$ perspective, the automobile industry programs the C/AV behavior in four planning stages: global route planning, behavior planning, motion planning, and local planning. To identify the state estimation and localization, $\mathrm{C} / \mathrm{AVs}$ are reinforced with numerous sensors for the perception. To model the performance of these sensors and the perception of the infrastructure environment, nanoscopic AV simulation tools are available. Nanoscopic AV simulation tools offer numerous customization options, including detailed maps, 3D vehicle models, sensors, vehicular physics-based models, and external programming interfaces for controlling the actors during the run-time. To better understand the nanoscopic AV simulation tools, a review analysis was performed, and the results are summarized in Table 5.

From the analysis, it is observed that more than thirty AV simulation tools are available in the market. They are commercial and open source in nature and are built using game engines [122, 123]. In general, game engines give the flexibility to place the actors over the $3 \mathrm{D}$ space. Based on their character, the behavior will be exhibited. By connecting the actors and agents, the simulation environment is built. The open-source unreal game engine [124] was found to be a popular game engine for building the simulation environment. Most simulation tools offer all kinds of basic sensors for modeling the AV perception. To control the actors during the simulation run-time, application programming interfaces are provided, mostly in python and MATLAB/ Simulink.

Interestingly most of these tools offer a cosimulation environment, in which different AV simulation tools are coupled with traffic microsimulation tools. During the cosimulation, the $\mathrm{C} / \mathrm{AV}$ behavior is completely governed by the AV simulation tool, where the behavior of C/AVs is modeled with perception from the system, and the entire traffic behavior is governed by the traffic microsimulation tool. These cosimulation strategies help in simulating the $\mathrm{C} /$ AVs more accurately and improve the simulation to achieve a more realistic estimation of the traffic operation. The review analysis observed that SUMO and VISSIM are used more often among other traffic microsimulation platforms as cosimulation tools. Given the open-source nature, SUMO is favored in the $\mathrm{AV}$ cosimulations.

The literature related to the C/AV cosimulation studies was reviewed and is summarized in Figure 8 and Table 6. The review analysis revealed that the studies are limited in nature. The cosimulation studies picked up recently, and a major proportion of these studies focused on developing methodologies for the $\mathrm{C} / \mathrm{AVs}$ cosimulation. Interestingly, researchers favored VISSIM to couple it with CarMaker and SUMO with Carla to carry the cosimulation.

The cosimulation research studies highly concentrated at local and motion planning of the C/AVs, and less weightage was given to the global planning. It can be noted that $\mathrm{AV}$ simulation tools use the detailed network and simultaneously couple it to the microsimulation which demands good computational power. During the cosimulation, the microsimulation runs are carried out by the externalities, reducing the simulation processing speed and impacting the run-time. Most AV simulation platforms provide inbuilt maps/networks; however, generating such detailed maps for the study section requires additional tools. Given this, AV cosimulation studies are highly dependent on the inbuilt maps/networks in the present context. Finally, currently the cosimulation is restricted to traffic volume, simulation time, and network size.

\section{Summary}

Given the long research history and research activities, traffic microsimulation has evolved as a core area in traffic engineering. The research activities in the traffic microsimulation domain were found to be highly dynamic in nature with numerous traffic microsimulation studies emerging across the world. Therefore, understanding the flow of the research can help in carrying future research studies. Thus, this study attempted to understand the literature in the traffic microsimulation research domain. Initially, the literature was mined, and the analysis, in terms of the source of microsimulation studies, was performed. To realize this, the involved institutes and the scientific journals were reviewed. From the review analysis, it was observed that worldwide researchers addressed the traffic problems considering the unique traffic conditions in their own countries. Based on the analysis, it was found that few institutes from certain countries are heavily involved in this domain. Traffic 


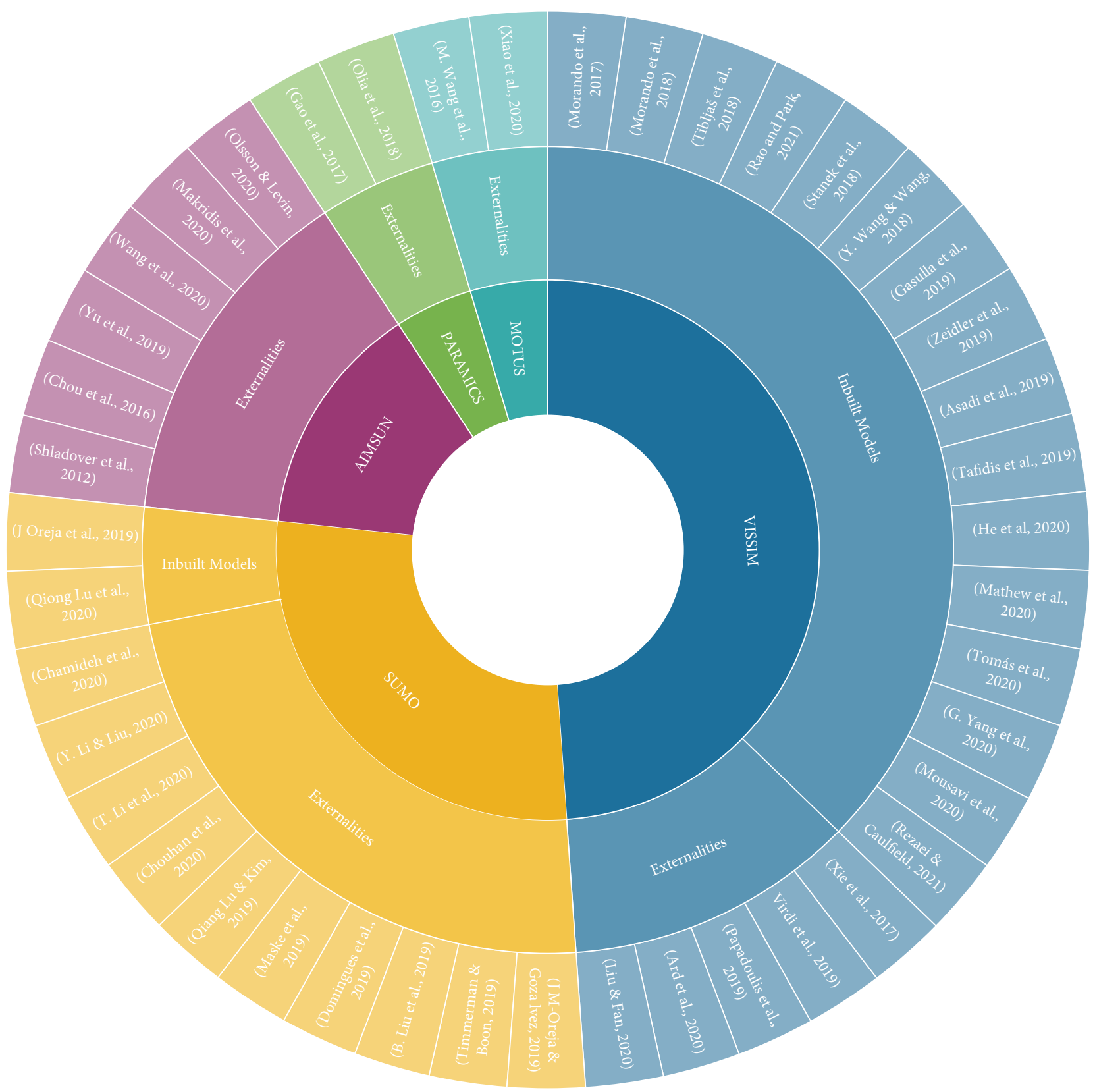

Figure 7: AV microsimulation studies over different platforms.

microsimulation studies have found a place in more than 50 journals and numerous conferences. Among them, some specific journals gathered a significant share of simulation studies.

For supporting the research activities and industrial applications, numerous traffic microsimulation platforms have evolved. In this paper, commercial and open-source microsimulation platforms were reviewed; from the review analysis, it was found that some specific platforms kept on evolving, while some others lost their share. Particularly in the previous decade, microsimulation studies have picked up the pace. As a result, a great number of microsimulation studies were conducted. Microsimulation platforms were upgraded over time to match the realistic field conditions as closely as possible. In the present context, microsimulation tools offer various customization options. To a certain extent, researchers can override the inbuilt models and test their frameworks, including advanced mathematical concepts, such as Artificial Intelligence logic.

Despite the many advantages, there are certain limitations associated with microsimulation platforms. The calibration of the simulation models plays a significant role in having confidence in the simulation outcomes. Given the sophisticated inbuilt models, microsimulation platforms demand detailed empirical data regarding the vehicle trajectories from field conditions. At the same time, generating such kind of microscopic data may not be viable all the time. Due to this, the simulations are calibrated with certain assumptions, and as a result, the same assumptions will be reflected in the simulation outcomes. While performing the 


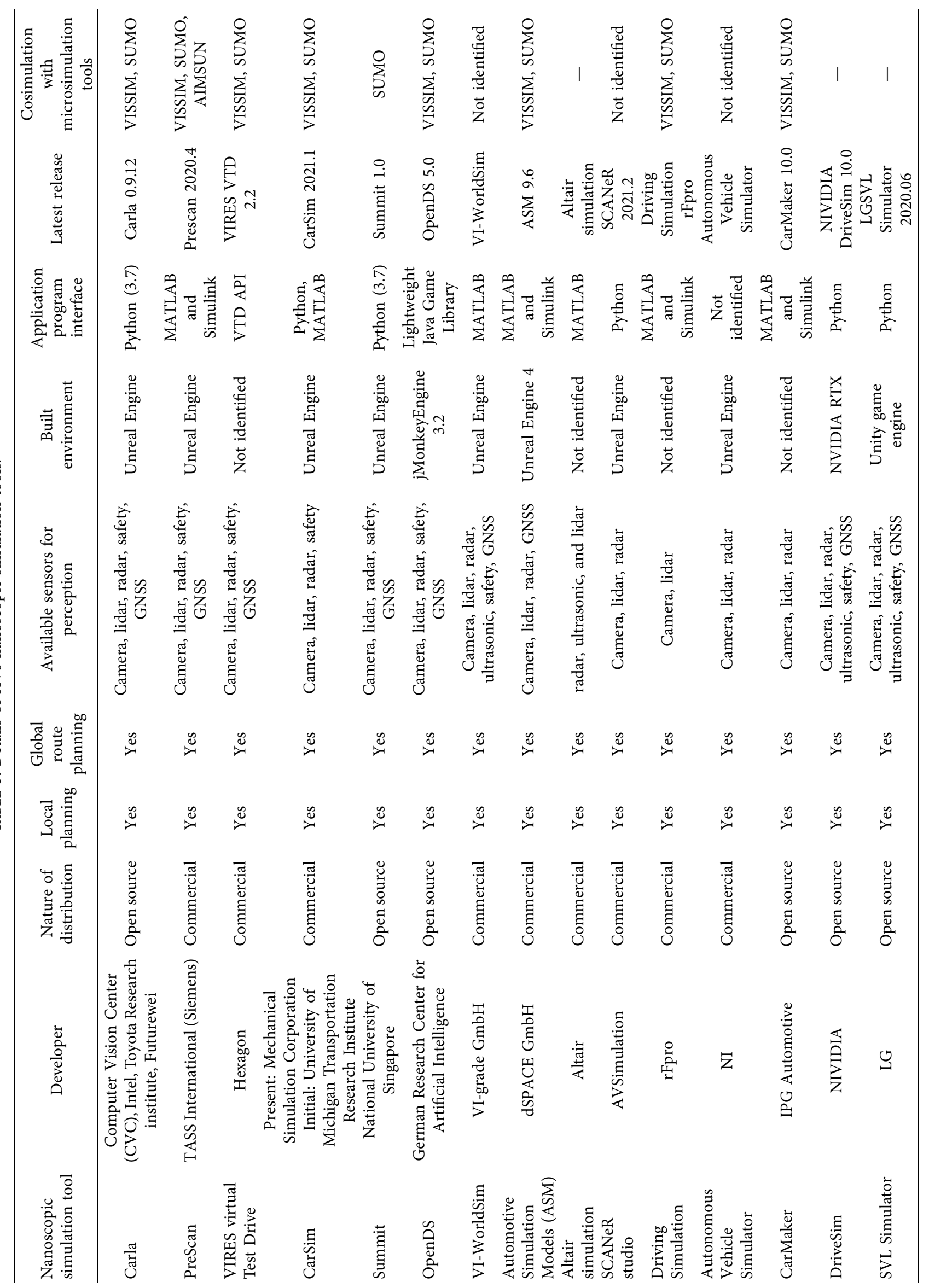




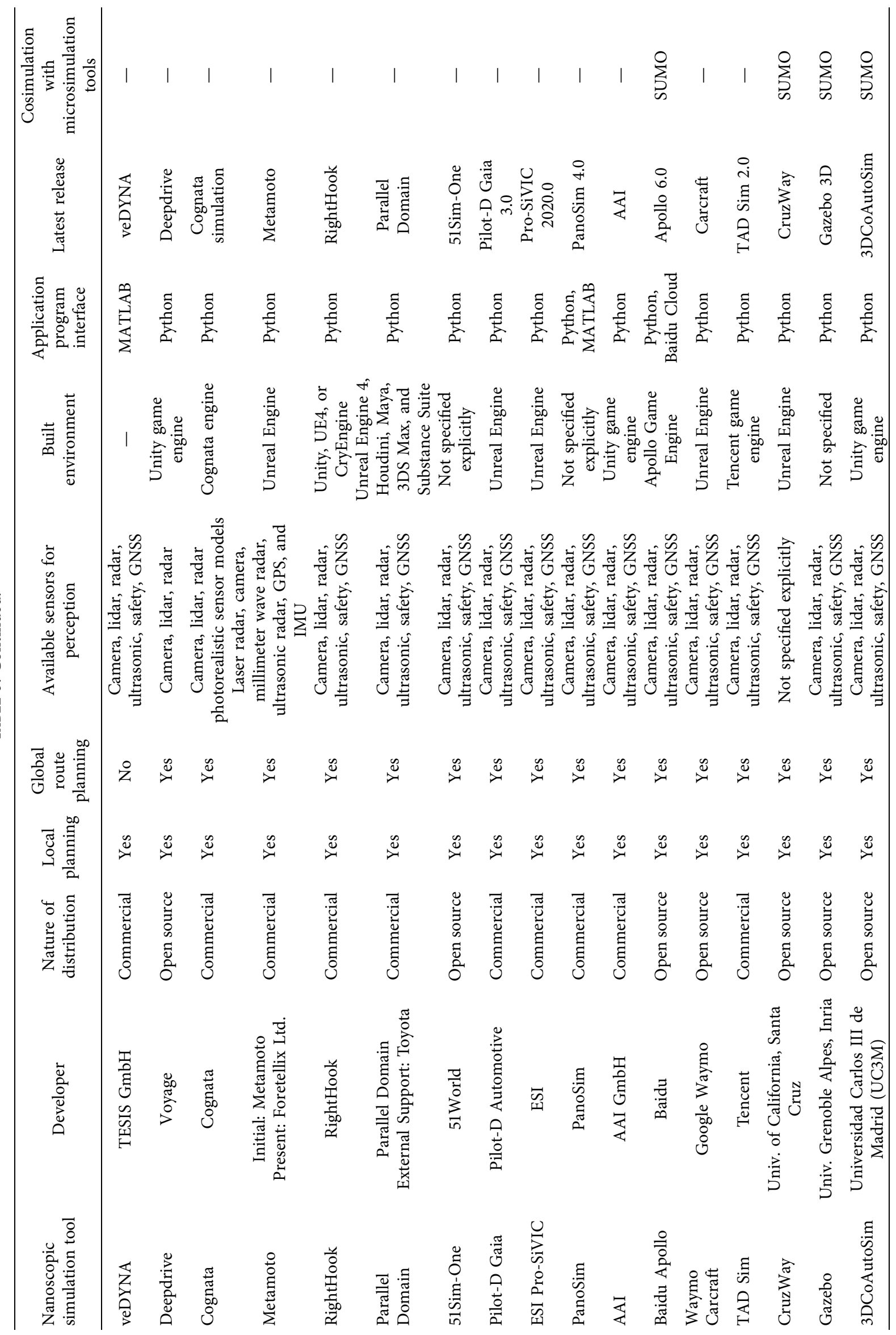




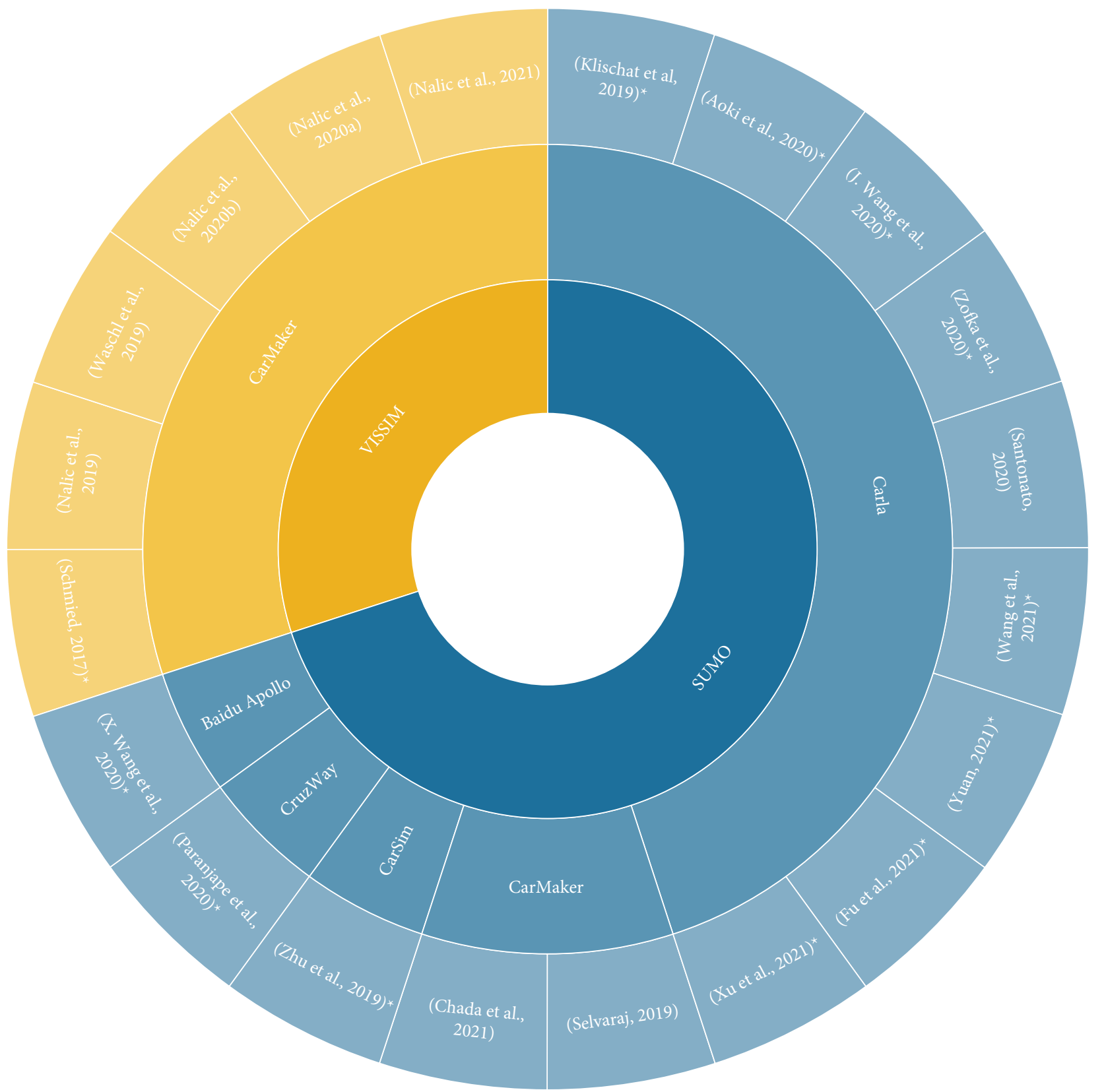

FIgURE 8: AV cosimulation studies over different platforms.

simulation runs with externalities, the processing falls to a single core in the computer processor in some microsimulation platforms. As a result, for medium-scale networks with heavy traffic flow conditions, the simulation run can overload a single processor's core, leading to simulation crashing. Presently, this is a limitation in expanding microsimulation studies with externalities for a more extensive network with heavy traffic.

\section{Future Prospects for AVS}

Given the considerable innovations in automobiles and top multinational conglomerates' involvement in research and innovation in this sector, anticipating AVs on our road network is becoming a realistic scenario for the near future. Additionally, this has brought new challenges for the researchers in the transportation domain. Traffic microsimulation platforms can be one of the dependable resources in understanding AVs' impacts from a traffic engineering point of view. Particularly in the second half of the last decade, researchers focused heavily on understanding AVs' effects on traffic performance. The literature examination shows that the research attempts in this direction have taken two significant approaches. According to the first approach, the researchers have used the inbuilt models from the simulation platforms and have adapted the model's parameters to replicate the behavior of AVs. The second approach used by researchers is coding the CAVs' behavior 


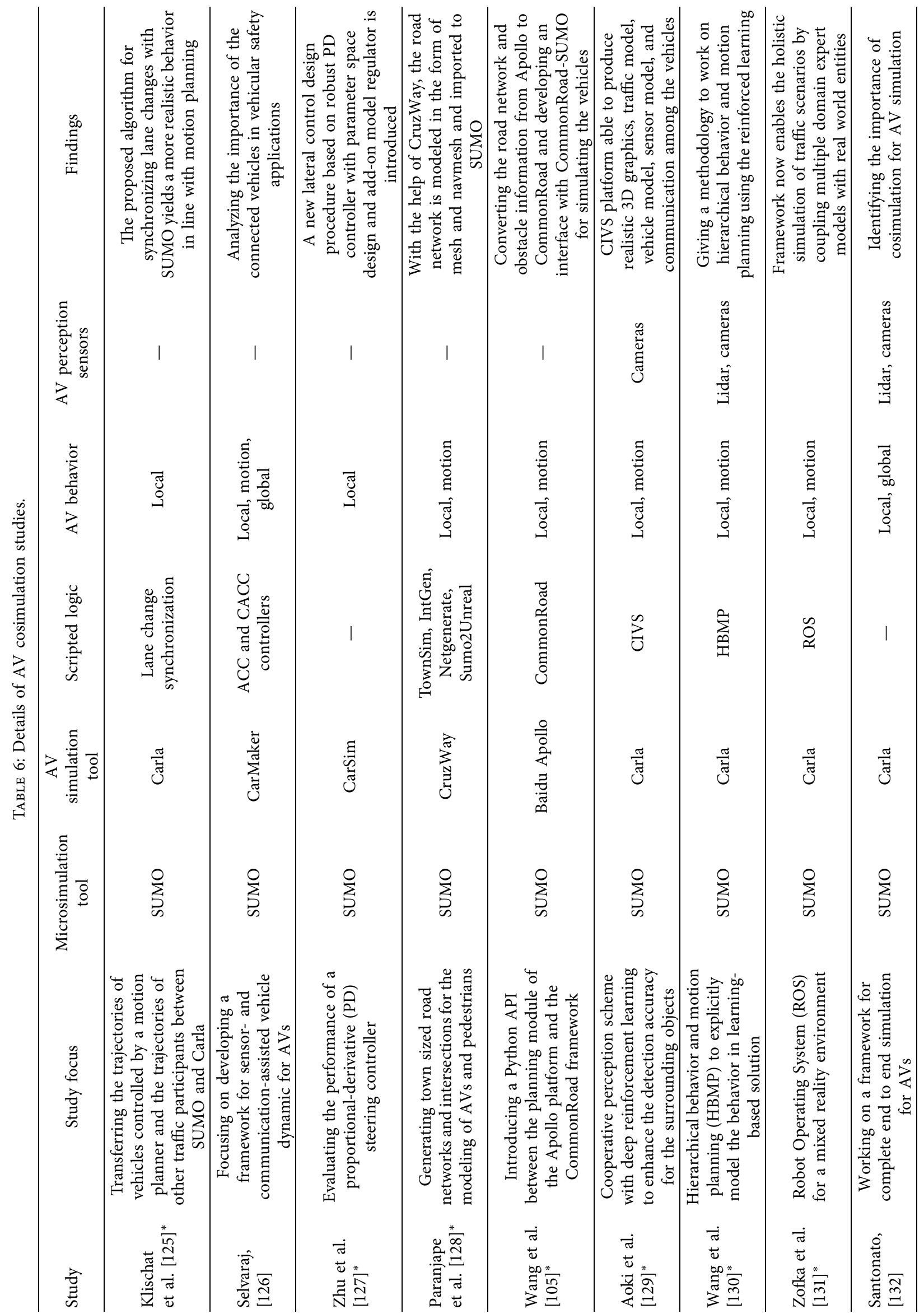




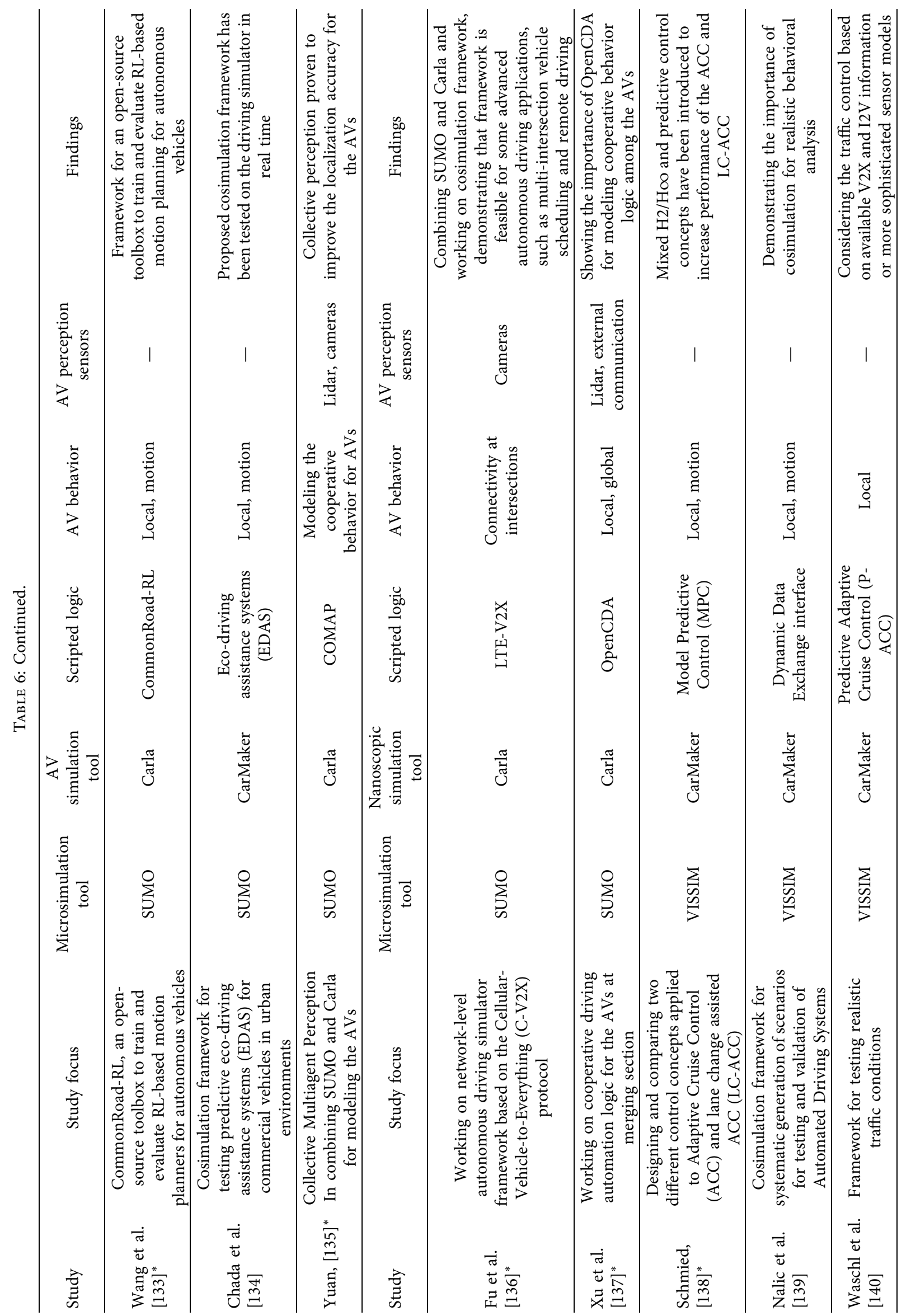




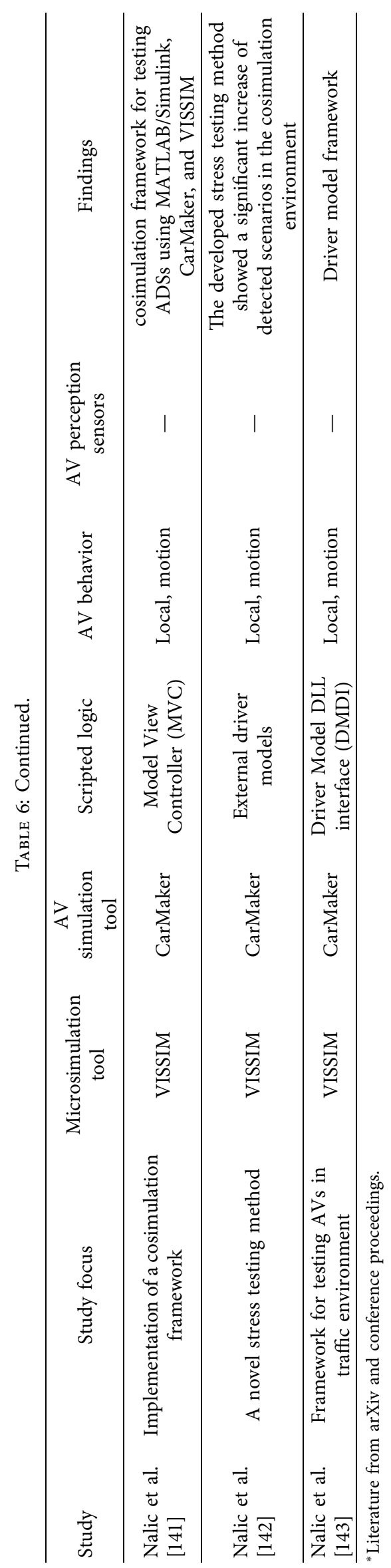


with the help of externalities for the simulation runs. Presently, both strategies have their advantages and limitations. Most studies have focused on the impacts of AVs penetration rates on safety and efficiency, with the majority predicting that, at low AV penetration rates, both safety and efficiency will be degraded. At higher AV penetration rates, the studies forecast significant improvements in safety and efficiency standards. It is inferred that the results across the different studies are not consistent; the AV transition penetration rates are varying across the studies and range from $20 \%$ to $70 \%$. The results of these studies are highly dependent on the inbuilt models of the simulation platforms. On the other hand, with externalities, researchers focused on the communication aspects among CAVs and worked on platooning models at midblock sections, cooperative merging models at merging sections, and cooperative gap formation models at intersections. The present ongoing studies adapt the behavioral model parameters in such a manner that C/AVs will have smooth behavior in comparison to human-driven vehicles. At the same time, the calibration of the parameters for C/AVs demands trajectory data of C/AVs from field conditions, which is limited in nature.

From the studies, it is concluded that most studies assume fully functional AVs in the simulation models. However, from a practical perspective, the functionality of $\mathrm{AVs}$ is discretized into five levels by the Society of $\mathrm{Au}-$ tomotive Engineers (SAE). In supporting this, presently, AVs of initial levels find their place on our road networks. As a result, there can be a mixed proportion with different $\mathrm{AV}$ levels that can be observed. In existing studies, not much weight is given to these aspects. This forms a huge research gap in the present AV microsimulation studies. From recent investigations, it is observed that AVs' presence can influence the behavior of human-driven vehicles. However, present microsimulation studies assume that human-driven vehicles will have the same usual interaction standards even with AVs, ignoring human behavioral adaption. This is once again one of the limitations of present AV microsimulation studies, which can be addressed by future studies.

Considering the importance of $\mathrm{C} / \mathrm{AV}$ motion planning in microsimulation, cosimulation frameworks have evolved in recent years. Cosimulation strategies show a good promise to model the C/AV behavior in a much-detailed manner combined with sensor perception. In the present context, most of the cosimulation studies are at initial levels and are highly focused on developing cosimulation frameworks. Given the computational constraint in cosimulation APIs, the cosimulation studies focus on a single vehicle with limited traffic volume and network. Cosimulation studies are highly focused on $\mathrm{C} / \mathrm{AV}$ motion planning with less weightage to traffic characteristics as in microsimulation. However, with the ongoing attempts and cosimulation APIs, it can be extended with a higher number of C/AVs guided based on the sensors in a traffic stream and the humandriven vehicles with the behavioral models. These developments will help develop new and improved strategies for better management of mixed traffic. At the same time, the results can build enough confidence for practitioners in accepting the research outcomes.

\section{Data Availability}

The data that support the findings of this study can be made available upon request.

\section{Conflicts of Interest}

The authors declare that there are no conflicts of interest regarding the publication of this paper.

\section{Acknowledgments}

This work was supported by the Applied and Technical Sciences (TTW), a subdomain of the Dutch Institute for Scientific Research (NWO), through the Project Safe and Efficient Operation of Automated and Human-Driven Vehicles in Mixed Traffic (SAMEN) under Contract 17187.

\section{References}

[1] S. Singh, "Critical reasons for crashes investigated in the national motor vehicle crash causation survey," National Center for Statistics and Analysis, National Highway Traffic Safety Administration, Washington, DC, USA, 2015.

[2] D. J. Fagnant and K. Kockelman, "Preparing a nation for autonomous vehicles: opportunities, barriers and policy recommendations," Transportation Research Part A: Policy and Practice, vol. 77, 2015.

[3] H. Liu, X. D. Kan, S. E. Shladover, X. Y. Lu, and R. E. Ferlis, "Impact of cooperative adaptive cruise control on multilane freeway merge capacity," Journal of Intelligent Transportation Systems: Technology, Planning, and Operations, vol. 22, no. 3, pp. 263-275, 2018.

[4] F. V. Webster, "Traffic signal settings," Road Res. Lab., London, UK, Road Research Technical Paper. No. 39, 1958.

[5] A. S. Kenyon and D. P. Morton, "Stochastic vehicle routing with random travel times," Transportation Science, vol. 37, no. 1, 2003.

[6] S. H. Stroeve, H. A. P. Blom, and G. J. Bakker, "Systemic accident risk assessment in air traffic by Monte Carlo simulation," Safety Science, vol. 47, no. 2, pp. 238-249, 2009.

[7] E. Veach, Robust Monte Carlo Methods for Light Transport Simulation, Diss. Dep. Comput. Sci. Stanford Univ, Stanford, CA, USA, 1997.

[8] S. F. Hafstein, R. Chrobok, A. Pottmeier, M. Schreckenberg, and F. C. Mazur, "A high-resolution cellular automata traffic simulation model with application in a freeway traffic information system," Computer-Aided Civil and Infrastructure Engineering, vol. 19, no. 5, pp. 338-350, 2004.

[9] M. E. Lrraga and L. Alvarez-Icaza, "Cellular automaton model for traffic flow based on safe driving policies and human reactions," Physica A: Statistical Mechanics and its Applications, vol. 389, no. 23, pp. 5425-5438, 2010.

[10] M. Saifuzzaman and Z. Zheng, "Incorporating human-factors in car-following models: a review of recent developments and research needs," Transportation Research Part C: Emerging Technologies, vol. 48, pp. 379-403, 2014.

[11] Z. Zheng, "Recent developments and research needs in modeling lane changing," Transportation Research Part B: Methodological, vol. 60, pp. 16-32, 2014. 
[12] R. Dowling, A. Skabardonis, and V. Alexiadis, "Traffic analysis toolbox volume III: guidelines for applying traffic microsimulation modeling software," Rep. No. FHWAHRT-04-040, U.S. DOT, Fed, Highw. Adm, Washington, DC, USA, 2004.

[13] Y. Hollander and R. Liu, "The principles of calibrating traffic microsimulation models," Transportation, vol. 35, pp. 347$362,2008$.

[14] J. J. Olstam and A. Tapani, "Comparison of car-following models," Swedish National Road and Transport Research Institute, Linköping, Sweden, 2004.

[15] J. A. Laval and C. F. Daganzo, "Lane-changing in traffic streams," Transportation Research Part B: Methodological, vol. 40, no. 3, pp. 251-264, 2006.

[16] D. De Jong, V. L. Knoop, and S. P. Hoogendoorn, "The effect of signal settings on the macroscopic fundamental diagram and its applicability in traffic signal driven perimeter control strategies," in Proceedings of the IEEE Conference on Intelligent Transportation Systems, Proceedings, ITSC, The Hague, The Netherlands, 2013.

[17] V. Astarita, V. Giofré, G. Guido, and A. Vitale, "Investigating road safety issues through a microsimulation model," in Procedia-Social and Behavioral Sciences, vol. 20, pp. 226-235, 2011.

[18] G. Song, L. Yu, and Y. Zhang, "Applicability of traffic microsimulation models in vehicle emissions estimates," Transportation Research Record: Journal of the Transportation Research Board, vol. 2270, no. 1, pp. 132-141, 2012.

[19] S. P. Hoogendoorn, M. Hauser, and N. Rodrigues, "Applying microscopic pedestrian flow simulation to railway station design evaluation in Lisbon, Portugal," Transportation Research Record: Journal of the Transportation Research Board, vol. 1878, no. 1, pp. 83-94, 2004.

[20] G. A. Klunder, H. Taale, L. Kester, and S. Hoogendoorn, "The effect of inaccurate traffic data for ramp metering: comparing loop detectors and cameras using information utility," IFAC Proceedings Volumes, vol. 47, no. 3, pp. 11318-11325, 2014.

[21] H. Taale, S. Hoogendoorn, and P. Legius, "Metering with traffic signal control - development and evaluation of an algorithm," Transportation Research Procedia, vol. 8, pp. 204-214, 2015.

[22] V. Astarita, G. Guido, A. Vitale, and V. Gallelli, "Analysis of non-conventional roundabouts performances through $\mathrm{mi}-$ croscopic traffic simulation," Applied Mechanics and Materials, vol. 505-506, pp. 481-488, 2014.

[23] M. S. Bains, S. S. Arkatkar, K. S. Anbumani, and S. Subramaniam, "Optimizing and modeling tollway operations using microsimulation," Journal of the Transportation Research Board, vol. 2615, no. 1, pp. 43-54, 2017.

[24] N. Raju, A. Jain, S. S. Arkatkar, and G. Joshi, "Experimental design for measuring operational performance of truck parking terminal using simulation technique," in Proceedings of the 98th Annual Meeting Transportation Research Board, Washington, DC, USA, 2019.

[25] Elsevier, Scopus Preview, Elsevier, Amsterdam, Netherlands, 2021, https://www.scopus.com.

[26] R. Balakrishna, C. Antoniou, M. Ben-Akiva, H. N. Koutsopoulos, and Y. Wen, "Calibration of microscopic traffic simulation models: methods and application," Transportation Research Record: Journal of the Transportation Research Board, vol. 1999, no. 1, pp. 198-207, 2007.

[27] L. Chu, H. X. Liu, J. S. Oh, and W. Recker, "A calibration procedure for microscopic traffic simulation," in Proceedings of the IEEE Conference on Intelligent Transportation Systems, Proceedings, ITSC, Shanghai, China, October 2003.

[28] V. P. Giofrè, M. Maciejewski, A. Merkisz-Guranowska, B. Piątkowski, and V. Astarita, "Real road network application of a new microsimulation tool: TRITONE," Arch Transport, vol. 27, no. 3, pp. 111-121, 2013.

[29] J. Olstam and A. Tapani, "A review of guidelines for applying traffic simulation to level-of-service analysis," in ProcediaSocial and Behavioral Sciences, vol. 16, pp. 771-780, 2011.

[30] T. Toledo, M. E. Ben-Akiva, D. Darda, M. Jha, and H. N. Koutsopoulos, "Calibration of microscopic traffic simulation models with aggregate data," Transportation Research Record: Journal of the Transportation Research Board, vol. 1876, no. 1, pp. 10-19, 2004.

[31] J. Schmidhuber, ROBOT CARS-Autonomous Vehicles-History of Self-Driving Cars-Best Robot Car, 2011, https://people. idsia.ch/ juergen/robotcars.html.

[32] M. A. Turk, D. G. Morgenthaler, K. D. Gremban, and M. Marra, "VITS-A vision system for autonomous land vehicle navigation," IEEE Transactions on Pattern Analysis and Machine Intelligence, vol. 10, pp. 342-361, 1988.

[33] R. Wallace, A. Stentz, C. Thorpe, H. Moravec, W. Whittaker, and T. Kanade, "First results in robot road following," Comput. Eng. Proc. Int. Comput. Eng. Conf, vol. 1, pp. 381387, 1985.

[34] cbinsights, 40+ Corporations Working on Autonomous Vehicles, 2020, https://www.cbinsights.com/research/autonomousdriverless-vehicles-corporations-list/.

[35] N. Adnan, S. Md Nordin, M. A. bin Bahruddin, and M. Ali, "How trust can drive forward the user acceptance to the technology? in-vehicle technology for autonomous vehicle," Transportation Research Part A: Policy and Practice, vol. 118, 2018.

[36] R. Drummond and Y. Zheng, Impact of Disturbances on Mixed Traffic Control with Autonomous Vehicles, arXiv, 2020.

[37] R. E. Stern, S. Cui, M. L. Delle Monache et al., "Dissipation of stop-and-go waves via control of autonomous vehicles: field experiments," Transportation Research Part C: Emerging Technologies, vol. 89, pp. 205-221, 2018.

[38] G. D. B. Cameron and G. I. D. Duncan, "Paramics-parallel microscopic simulation of road traffic," The Journal of Supercomputing, vol. 10, pp. 25-53, 1996.

[39] H. Fritzsche, "A model for traffic simulation," Traffic Engineering and Control, vol. 35, no. 5, 1994.

[40] Paramics, PARAMICS 3D Microsimulation, https://www. paramics.co.uk/en/about-us/article/paramics-microsimulation, 2021.

[41] R. Wiedemann, Simulation des Straßenverkehrsflusses. Schriftenreihe des If $\mathrm{V}$, Institut für Verkehrswesen. Universität Karlsruhe, vol. 8, 1974.

[42] M. Ben-Akiva, D. Cuneo, M. Hasan, M. Jha, and Q. Yang, "Evaluation of freeway control using a microscopic simulation laboratory," Transportation Research Part C: Emerging Technologies, vol. 11, no. 1, pp. 29-50, 2003.

[43] Q. Yang and H. N. Koutsopoulos, "A microscopic traffic simulator for evaluation of dynamic traffic management systems," Transportation Research Part C: Emerging Technologies, vol. 4, no. 3, pp. 113-129, 1996.

[44] J. Barceló, E. Codina, J. Casas, J. L. Ferrer, and D. García, "Microscopic traffic simulation: a tool for the design, analysis and evaluation of intelligent transport systems," Journal of Intelligent and Robotic Systems: Theory and Applications, vol. 41, pp. 173-203, 2005. 
[45] B. Van Arem, C. J. G. Van Driel, and R. Visser, "The impact of cooperative adaptive cruise control on traffic-flow characteristics," IEEE Transactions on Intelligent Transportation Systems, vol. 7, no. 4, pp. 429-436, 2006.

[46] A. Halati, H. Lieu, and S. Walker, "CORSIM- corridor traffic simulation model," in Proceedings of the Conference on Traffic Congestion and Traffic Safety in the 21st Century, Chicago, IL, USA, 1997.

[47] L. E. Owen, Y. Zhang, L. Rao, and G. McHale, "Traffic flow simulation using CORSIM," in Proceedings of the Winter Simulation Conference, Orlando, FL, USA, December 2000.

[48] M. Behrisch, L. Bieker, J. Erdmann, and D. Krajzewicz, SUMO-Simulation of Urban MObility, Iaria, 2011.

[49] D. Krajzewicz and G. Hertkorn, "SUMO (Simulation of Urban MObility) an open-source traffic simulation," in Proceedings of the 4th Middle East Symposium on Simulation and Modelling (MESM20002), Sharjah. UAE, pp. 183-187, 2000.

[50] D. Jiḿenez, F. Mũnoz, S. Arias, and J. Hincapie, "Software for calibration of transmodeler traffic microsimulation models," in Proceedings of the IEEE Conference on Intelligent Transportation Systems, Proceedings, ITSC, Rio de Janeiro, Brazil, 2016.

[51] L. Lu, T. Yun, L. Li, Y. Su, and D. Yao, "A comparison of phase transitions produced by PARAMICS, TransModeler, and VISSIM," IEEE Intelligent Transportation Systems Magazine, vol. 2, no. 3, pp. 19-24, 2010.

[52] MOTUS, MOTUS_Microscopic Open Traffic Simulation, 2018.

[53] W. Schakel, Development, Simulation and Evaluation of InCar Advice on Headway, Speed and Lane, 2015.

[54] H. Van Lint, W. Schakel, G. Tamminga, P. Knoppers, and A. Verbraeck, "Getting the human factor into traffic flow models: new open-source design to simulate next generation of traffic operations," Transportation Research Record: Journal of the Transportation Research Board, vol. 2561, no. 1, pp. 25-33, 2016.

[55] M. V. Aerde, B. Hellinga, M. Baker, and H. Rakha, "Integration: an overview of traffic simulation features," in Proceedings of the Transportation Research Board Annual Meet, Washington, DC, USA, 1996.

[56] K. Shaaban and I. Kim, "Comparison of SimTraffic and VISSIM microscopic traffic simulation tools in modeling roundabouts," Procedia Computer Science, vol. 52, pp. 43-50, 2015.

[57] I. Kosonen, Hutsim-Urban Traffic Simulation and Control Model: Principles and Applications, HELSINKI Univ. Technol. Transp. Eng. Publ., Espoo, Finland, 1999.

[58] P. T. R. Wang and W. P. Niedringhaus, "Distributed/parallel traffic simulation for IVHS applications," in Proceedings of the Winter Simulation Conference Proceedings, Los Angeles, CA, USA, 1993.

[59] A. Stevanovic and N. Mitrovic, "Traffic microsimulation for flexible utilization of urban roadways," Transportation Research Record: Journal of the Transportation Research Board, vol. 2673, pp. 92-104, 2019.

[60] T. Dijker, FOSIM (Freeway Operations SIMulation), 2002.

[61] A. Tapani, A Traffic Simulation Modeling Framework for Rural Highways, 2005.

[62] M. Camurri, M. Mamei, and F. Zambonelli, "Urban traffic control with co-fields," in Proceedings of the Environments for Multi-Agent Systems III, Third International Workshop, E4MAS, pp. 239-253, Hakodate, Japan, 2007.
[63] M. Maciejewski and J. Bischoff, "Congestion effects of autonomous taxi fleets," Transport, vol. 33, 2018.

[64] A. T. Moreno, A. Michalski, C. Llorca, and R. Moeckel, "Shared autonomous vehicles effect on vehicle-km traveled and average trip duration," Journal of Advanced Transportation, vol. 2018, Article ID 8969353, 2018.

[65] J. Hudson, M. Orviska, and J. Hunady, "People's attitudes to autonomous vehicles," Transportation Research Part A: Policy and Practice, vol. 121, pp. 164-176, 2019.

[66] A. O. Salonen and N. Haavisto, "Towards autonomous transportation. passengers' experiences, perceptions and feelings in a driverless shuttle bus in Finland," Sustainable Times, vol. 11, no. 3, p. 588, 2019.

[67] S. Nordhoff, J. de Winter, R. Madigan, N. Merat, B. van Arem, and R. Happee, "User acceptance of automated shuttles in Berlin-Schöneberg: a questionnaire study," Transportation Research Part F: Traffic Psychology and Behaviour, vol. 58, pp. 843-854, 2018.

[68] P. Koopman and M. Wagner, "Challenges in autonomous vehicle testing and validation," SAE International Journal of Transportation Safety, vol. 4, no. 1, pp. 15-24, 2016.

[69] S. D. Pendleton, H. Andersen, X. Du et al., "Perception, planning, control, and coordination for autonomous vehicles," Machines, vol. 5, no. 1, p. 6, 2017.

[70] Y. Xie, H. Zhang, N. H. Gartner, and T. Arsava, "Collaborative merging strategy for freeway ramp operations in a connected and autonomous vehicles environment," Journal of Intelligent Transportation Systems: Technology, Planning, and Operations, vol. 21, no. 2, 2017.

[71] V. Milanés and S. E. Shladover, "Modeling cooperative and autonomous adaptive cruise control dynamic responses using experimental data," Transportation Research Part C: Emerging Technologies, vol. 48, pp. 285-300, 2014.

[72] M. Wang, W. Daamen, S. P. Hoogendoorn, and B. Van Arem, "Cooperative car-following control: distributed algorithm and impact on moving Jam features," IEEE Transactions on Intelligent Transportation Systems, vol. 17, no. 5, 2016.

[73] N. J. Goodall, B. L. Smith, and B. Park, "Traffic signal control with connected vehicles," Journal of the Transportation Research Board, vol. 2381, no. 1, pp. 65-72, 2014.

[74] A. C. Mersky and C. Samaras, "Fuel economy testing of autonomous vehicles," Transportation Research Part C: Emerging Technologies, vol. 65, pp. 31-48, 2016.

[75] A. Papadoulis, M. Quddus, and M. Imprialou, "Evaluating the safety impact of connected and autonomous vehicles on motorways," Accident Analysis and Prevention, vol. 124, pp. 12-22, 2019.

[76] L. Ye and T. Yamamoto, "Modeling connected and autonomous vehicles in heterogeneous traffic flow," Physica A: Statistical Mechanics and its Applications, vol. 490, pp. 269-277, 2018.

[77] T. Hashimoto and H. Yanagisawa, "Risk feeling index of autonomous vehicle behavior," in Proceedings of the International Symposium on Affective Science and Engineering, Tokyo, Japan, 2020.

[78] D. K. M. Kufoalor, T. A. Johansen, E. F. Brekke, A. Hepsø, and K. Trnka, "Autonomous maritime collision avoidance: field verification of autonomous surface vehicle behavior in challenging scenarios," Journal of Robotics, vol. 37, 2020.

[79] M. M. Morando, Q. Tian, L. T. Truong, and H. L. Vu, "Studying the safety impact of autonomous vehicles using simulation-based surrogate safety measures," Journal of 
Advanced Transportation, vol. 2018, Article ID 6135183, 2018.

[80] M. M. Morando, L. T. Truong, and H. L. Vu, "Investigating safety impacts of autonomous vehicles using traffic microsimulation," in Proceedings of the ATRF 2017-Australasian Transport Research Forum 2017, Auckland, New Zealand, 2017.

[81] A. D. Tibljaš, T. Giuffrè, S. Surdonja, and S. Trubia, "Introduction of Autonomous Vehicles: roundabouts design and safety performance evaluation," Sustainable Times, vol. 10, no. 4, p. 1060, 2018.

[82] D. Stanek, E. Huang, R. T. Milam, and Y. A. Wang, "Measuring autonomous vehicle impacts on congested networks using simulation," in Proceedings of the Transportation Research Board 97th Annual Meeting, pp. 1-19, Washington DC, USA, 2018.

[83] Y. Wang and L. Wang, "Autonomous vehicles' performance on single lane road: a simulation under VISSIM environment," in Proceedings-2017 10th International Congress on Image and Signal Processing, BioMedical Engineering and Informatics, CISP-BMEI 2017, Shanghai, China, 2018.

[84] M. Martin-Gasulla, P. Sukennik, and J. Lohmiller, "Investigation of the impact on throughput of connected autonomous vehicles with headway based on the leading vehicle type," Transportation Research Record: Journal of the Transportation Research Board, vol. 2673, no. 5, pp. 617-626, 2019.

[85] F. E. Asadi, A. K. Anwar, and J. C. Miles, "Investigating the potential transportation impacts of connected and autonomous vehicles," in Proceedings of the 2019 IEEE ICCVE: The 8th IEEE International Conference on Connected Vehicles and Expo (ICCVE), Graz, Austria, 2019.

[86] S. He, S. He, X. Guo et al., "Freeway traffic speed estimation of mixed traffic using data from connected and autonomous vehicles with a low penetration rate," Journal of Advanced Transportation, vol. 2020, Article ID 1361583, 2020.

[87] S. Mathew, S. Duvvuri, and S. S. Pulugurtha, "Modeling and assessing the influence of connected and automated vehicle penetration rates on urban arterial road operational performance," in Proceedings of the International Conference on Transportation and Development 2020: Emerging Technologies and Their Impacts-Selected Papers from the International Conference on Transportation and Development, Reston, VA, USA, 2020.

[88] R. F. Tomás, P. Fernandes, E. MacEdo, J. M. Bandeira, and M. C. Coelho, "Assessing the emission impacts of autonomous vehicles on metropolitan freeways," Transportation Research Procedia, vol. 47, pp. 617-624, 2020.

[89] G. Yang, M. Ahmed, and E. Adomah, "An integrated microsimulation approach for safety performance assessment of the Wyoming connected vehicle pilot deployment program," Accident Analysis and Prevention, vol. 146, Article ID 105714, 2020.

[90] S. Maryam Mousavi, D. Lord, B. Dadashova, and S. Reza Mousavi, "Can Autonomous Vehicles Enhance Traffic Safety at Unsignalized Intersections?" in Proceedings of the International Conference on Transportation and Development, pp. 194-206, Reston, VA, USA, 2020.

[91] P. Tafidis, A. Pirdavani, T. Brijs, and H. Farah, "Intersection control type effect on automated vehicle operation," in Proceedings of the CICTP 2019: Transportation in Chin -Connecting the World-Proceedings of the 19th COTA International Conference of Transportation Professionals, Nanjing, China, 2019.
[92] A. Rezaei and B. Caulfield, "Simulating a transition to autonomous mobility," Simulation Modelling Practice and Theory, vol. 106, Article ID 102175, 2021.

[93] Q. Lu, T. Tettamanti, D. Hörcher, and I. Varga, “The impact of autonomous vehicles on urban traffic network capacity: an experimental analysis by microscopic traffic simulation," Transportation Letters, vol. 12, no. 8, pp. 540-549, 2020.

[94] J. Mena-Oreja, J. Gozalvez, and M. Sepulcre, "Effect of the configuration of platooning maneuvers on the traffic flow under mixed traffic scenarios," in Proceedings of the IEEE Vehicular Networking Conference, Los Angeles, CA, USA, December 2019.

[95] V. Zeidler, H. S. Buck, L. Kautzsch, P. D. P. Vortisch, and C. Weyland, "Simulation of autonomous vehicles based on Wiedemann's car following model in," in Proceedings of the Transportation Research Board 98th Annual Meeting, Washington, DC, USA, January 2019.

[96] R. S. Rao, S. Yoon. Park, and G. L. Chang, "Developing the guidelines for managing autonomous vehicle flows on congested highways: a case study of MD-100," Simulation: Transactions of the Society for Modeling and Simulation International, vol. 97, no. 6, pp. 367-382, 2021.

[97] W. Gao, Z. P. Jiang, and K. Ozbay, "Data-driven adaptive optimal control of connected vehicles," IEEE Transactions on Intelligent Transportation Systems, vol. 18, no. 5, 2017.

[98] A. Olia, S. Razavi, B. Abdulhai, and H. Abdelgawad, "Traffic capacity implications of automated vehicles mixed with regular vehicles," Journal of Intelligent Transportation Systems: Technology, Planning, and Operations, vol. 22, no. 3, 2018.

[99] N. Virdi, H. Grzybowska, S. T. Waller, and V. Dixit, "A safety assessment of mixed fleets with connected and autonomous vehicles using the surrogate safety assessment module," Accident Analysis and Prevention, vol. 131, pp. 95-111, 2019.

[100] T. Ard, R. A. Dollar, A. Vahidi, Y. Zhang, and D. Karbowski, "Microsimulation of energy and flow effects from optimal automated driving in mixed traffic," Transportation Research Part C: Emerging Technologies, vol. 120, 2020.

[101] P. Liu and W. Fan, "Exploring the impact of connected and autonomous vehicles on freeway capacity using a revised intelligent driver model," Transportation planning and technology, vol. 43, no. 3, pp. 279-292, 2020.

[102] S. E. Shladover, D. Su, and X. Y. Lu, "Impacts of cooperative adaptive cruise control on freeway traffic flow," Transportation Research Record, vol. 2324, no. 1, pp. 63-70, 2012.

[103] F. C. Chou, S. E. Shladover, and G. Bansal, "Coordinated merge control based on V2V communication," in Proceedings of the IEEE Vehicular Networking Conference (VNC), Columbus, OH, USA, October 2016.

[104] H. Yu, S. Tak, M. Park, and H. Yeo, "Impact of autonomousvehicle-only lanes in mixed traffic conditions," Transportation Research Record: Journal of the Transportation Research Board, vol. 2673, pp. 430-439, 2019.

[105] X. Wang, A.-K. Rettinger, and M. Althoff, "Coupling apollo with the CommonRoad motion planning framework," FISITA World Congress, pp. 1-8, 2020.

[106] M. Makridis, K. Mattas, C. Mogno, B. Ciuffo, and G. Fontaras, "The impact of automation and connectivity on traffic flow and $\mathrm{CO}_{2}$ emissions. A detailed microsimulation study," Atmospheric Environment, vol. 226, Article ID 117399, 2020.

[107] J. Olsson and M. W. Levin, "Integration of microsimulation and optimized autonomous intersection management," 
Journal of Transportation Engineering, Part A: Systems, vol. 146, no. 9, 2020.

[108] J. Mena-Oreja and J. Gozalvez, "Permit-SUMO simulator for platooning maneuvers in mixed traffic scenarios," in Proceedings of the IEEE Conference on Intelligent Transportation Systems, Proceedings, ITSC, Maui, HI, USA, September 2018.

[109] R. W. Timmerman and M. A. A. Boon, "Platoon forming algorithms for intelligent street intersections," Transportmetrica A: Transport Science, vol. 17, no. 3, pp. 278-307, 2019.

[110] B. Liu, Q. Shi, Z. Song, and A. El Kamel, "Trajectory planning for autonomous intersection management of connected vehicles," Simulation Modelling Practice and Theory, 2019.

[111] G. Domingues, J. Cabral, J. Mota, P. Pontes, Z. Kokkinogenis, and R. J. Rossetti, "Traffic simulation of lane-merging of autonomous vehicles in the context of platooning," in Proceedings of the 2018 IEEE International Smart Cities Conference (ISC2), pp. 1-6, Kansas City, MO, USA, September 2018.

[112] H. Maske, T. Chu, and U. Kalabić, "Large-scale traffic control using autonomous vehicles and decentralized deep reinforcement learning," in Proceedings of the 2019 IEEE Intelligent Transportation Systems Conference (ITSC), pp. 3816-3821, Auckland, New Zealand, October 2019.

[113] Q. Lu and K. D. Kim, "Autonomous and connected intersection crossing traffic management using discrete-time occupancies trajectory," Applied Intelligence, vol. 49, no. 5, 2019.

[114] A. P. Chouhan, G. Banda, and K. Jothibasu, "A cooperative algorithm for lane sorting of autonomous vehicles," IEEE Access, vol. 8, 2020.

[115] T. Li, F. Guo, R. Krishnan, A. Sivakumar, and J. Polak, "Right-of-way reallocation for mixed flow of autonomous vehicles and human driven vehicles," Transportation Research Part C: Emerging Technologies, vol. 115, Article ID 102630, 2020.

[116] Y. Li and Q. Liu, "Intersection management for autonomous vehicles with vehicle-to-infrastructure communication," PLoS One, vol. 15, pp. 1-12, 2020.

[117] S. Chamideh, W. Tärneberg, and M. Kihl, "Centralized coordination of autonomous vehicles at intersections," in Proceedings of the 28th International Conference on Software, Telecommunications and Computer Networks (SoftCOM 2020), IEEE-Institute of Electrical and Electronics Engineers Inc., Hvar, Croatia, 2020.

[118] L. Xiao, M. Wang, and B. Van Arem, "Traffic flow impacts of converting an HOV lane into a dedicated CACC lane on a freeway corridor," IEEE Intelligent Transportation Systems Magazine, vol. 12, no. 1, pp. 60-73, 2020.

[119] P. G. Gipps, "A behavioural car-following model for computer simulation," Transportation Research Part B: Methodological, vol. 15, pp. 105-111, 1981.

[120] M. Treiber, A. Hennecke, and D. Helbing, "Congested traffic states in empirical observations and microscopic simulations," Physical Review E-Statistical Physics, Plasmas, Fluids, and Related Interdisciplinary Topics, vol. 62, pp. 1805-1824, 2000.

[121] M. Bando, K. Hasebe, A. Nakayama, A. Shibata, and Y. Sugiyama, "Dynamical model of traffic congestion and numerical simulation," Physical Review E-Statistical Physics, Plasmas, Fluids, and Related Interdisciplinary Topics, vol. 51, pp. 1035-1042, 1995.
[122] W. Li, C. W. Pan, R. Zhang et al., "AADS: augmented autonomous driving simulation using data-driven algorithms," Science Robotics, vol. 4, no. 28, 2019.

[123] C. Politowski, F. Petrillo, J. E. Montandon, M. T. Valente, and Y. G. Guéhéneuc, "Are game engines software frameworks? a three-perspective study," Journal of Systems and Software, vol. 171, 2021.

[124] V. Kushnir and B. Koman, "Creating AI for games with unreal engine 4," Electronic Journal of Information Technology, vol. 9, 2018.

[125] M. Klischat, O. Dragoi, M. Eissa, and M. Althoff, "Coupling SUMO with a motion planning framework for automated vehicles," SUMO User Conference 2019, vol. 62, pp. 1-9, 2019.

[126] D. C. Selvaraj, "Development of a framework for sensor- and communication- assisted vehicle dynamic," 2019.

[127] S. Zhu, S. Y. Gelbal, X. Li, M. R. Cantas, B. Aksun-Guvenc, and L. Guvenc, "Parameter space and model regulation based robust, scalable and replicable lateral control design for autonomous vehicles," in Proceedings of the IEEE Conference on Decision and Control, Nice, France, December 2019.

[128] I. Paranjape, A. Jawad, Y. Xu, A. Song, and J. Whitehead, “A modular architecture for procedural generation of towns, intersections and scenarios for testing autonomous vehicles," in Proceedings of the IEEE Intelligent Vehicles Symposium, Las Vegas, NV, USA, October 2020.

[129] S. Aoki, T. Higuchi, and O. Altintas, "Cooperative perception with deep reinforcement learning for connected vehicles," in Proceedings of the IEEE Intelligent Vehicles Symposium, Las Vegas, NV, USA, October 2020.

[130] J. Wang, Y. Wang, D. Zhang, Y. Yang, and R. Xiong, "Learning hierarchical behavior and motion planning for autonomous driving," in Proceedings of the IEEE International Conference on Intelligent Robots and Systems, Prague, Czech Republic, October 2020.

[131] M. R. Zofka, L. Tottel, M. Zipfl et al., "Pushing ROS towards the dark side: a ROS-based Co-simulation architecture for mixed-reality test systems for autonomous vehicles," in Proceedings of the IEEE International Conference on Multisensor Fusion and Integration for Intelligent Systems, September 2020.

[132] S. Santonato, A complete end-to-end simulation flow for autonomous driving frameworks, Doctoral dissertation, Politecnico di Torino, Turin, Italy.

[133] X. Wang, H. Krasowski, and M. Althoff, "CommonRoad-RL: a configurable reinforcement learning environment for motion planning of autonomous vehicles," in Proceedings of the IEEE International Conference on Intelligent Transportation Systems, Indianapolis, IN, USA, 2021.

[134] S. K. Chada, D. Görges, A. Ebert, and R. Teutsch, “A driverin-the-loop co-simulation framework for testing predictive EDAS for commercial vehicles in urban environments," Commercial Vehicle Technology, Springer Vieweg, Wiesbaden, Germany, 2021.

[135] Y. Yuan, Comap: A Synthetic Dataset for Collective MultiAgent Comap: a Synthetic Dataset for Collective Multi-Agent Perception, 2021.

[136] S. Fu, W. Zhang, and Z. Jiang, "A network-level connected autonomous driving evaluation platform implementing C-V2X technology," China Communications, vol. 18, 2021.

[137] R. Xu, Y. Guo, X. Han, X. Xia, H. Xiang, and J. Ma, "OpenCDA: an open cooperative driving automation framework integrated with co-simulation," 2021, https:// arxiv.org/abs/2107.06260. 
[138] R. Schmied, Mixed $H 2 / H$ and predictive adaptive cruise control: design, evaluation and comparison/eingereicht von Roman Schmied, Doctoral dissertation, Universität Linz, Linz, Austria, 2017.

[139] D. Nalic, A. Eichberger, G. Hanzl, M. Fellendorf, and B. Rogic, "Development of a co-simulation framework for systematic generation of scenarios for testing and validation of automated driving systems," in Proceedings of the 2019 IEEE Intelligent Transportation Systems Conference, ITSC 2019, Auckland, New Zealand, October 2019.

[140] H. Waschl, R. Schmied, D. Reischl, and M. Stolz, "A virtual development and evaluation framework for ADAS-case study of a P-ACC in a connected environment," in Control Strategies for Advanced Driver Assistance Systems and Autonomous Driving Functions, Lecture Notes in Control and Information Sciences, Springer, Cham. Switzerland, 2019.

[141] D. Nalic, A. Pandurevic, A. Eichberger, and B. Rogic, "Design and implementation of a co-simulation framework for testing of automated driving systems," Sustainable Times, vol. 12, 2020.

[142] D. Nalic, H. Li, A. Eichberger, C. Wellershaus, A. Pandurevic, and B. Rogic, "Stress testing method for scenario-based testing of automated driving systems," IEEE Access, vol. 8, 2020.

[143] D. Nalic, A. Pandurevic, A. Eichberger, M. Fellendorf, and B. Rogic, "Software framework for testing of automated driving systems in the traffic environment of vissim," Energies, vol. 14, 2021. 